\title{
sobre el funcionamiento de la viga-carril metálica y las tensiones locales y secundarias
}

JOSE RIVACOBA, Ingeniero de Caminos, Canales y Puertos Jefe de la Sección de Estructuras Metálicas de SENER

\begin{abstract}
sinopsis En la segunda parte del artículo se completa la información sobre la viga-carril $566-3$ de sección en doble te, presentada en la primera parte, y se comentan los métodos analíticos de cálculo de la viga-carril de sección en cajón.
\end{abstract}

\section{SEGUNDA PARTE}

\section{LAS TENSIONES LOCALES EN VIGAS-CARRILES DE SECCION EN DOBLE TE}

En la primera parte de este artículo ${ }^{1}$, persiguiendo la brevedad en la exposición, hemos ido, al parecer, demasiado lejos, descargando sobre el lector interesado la difícil tarea de conseguir información complementaria en trabajos publicados hace ya más de 10 años en universidades de la lejana Rusia. Con el afán de subsanar los inconvenientes de la citada brevedad empezamos esta segunda parte del artículo tratando sobre las tensiones locales, completando la información presentada en el artículo anterior.

En el caso concreto de la placa (el alma de la viga-carril) solicitada por esfuerzos cortantes uniformes $q_{1}=q_{2}=q$, en la que $a=b=l$, las expresiones de las funciones $\alpha_{3}, \beta_{3}$ y $\gamma_{3}$ obtenidas por Lampsi ${ }^{2}$ son las siguientes:

$$
\begin{aligned}
& \alpha_{3}=-\frac{2}{\pi} \cdot \int_{0}^{\infty}\left[H_{1}(z, \mu)+H_{2}(z, \mu)\right] \cdot(1-\cos z \eta) \cdot \cos z \zeta \cdot \frac{d z}{z} ; \\
& \beta_{3}=\frac{2}{\pi} \cdot \int_{0}^{\infty}\left[H_{3}(z, \mu)+H_{4}(z, \mu)\right] \cdot(1-\cos z \eta) \cdot \cos z \zeta \cdot \frac{d z}{z} ; \\
& \gamma_{3}=-\frac{2}{\pi} \cdot \int_{0}^{\infty}\left[H_{5}(z, \mu)+H_{6}(z, \mu)\right] \cdot(1-\cos z \eta) \cdot \operatorname{sen} z \zeta \cdot \frac{d z}{z} ;
\end{aligned}
$$

en las que se ha conservado la misma notación:

$$
\zeta=\frac{x}{h} ; \quad \mu=\frac{y}{h} ; \eta=\frac{l}{h} ; z=m \cdot h
$$

NOTA: Debemos advertir al lector que la [1] de la ${ }^{1}$ es la expresión de la intensidad de la presión, y que en la página 73, en la cuarta línea, desde arriba, se debe sustituir la $\xi$ por una $\zeta$. Además, la [21] expresa el valor de la parte del momento torsor exterior soportada por el conjunto carril-cabeza superior de la viga, que será soportada en su totalidad por la cabeza sola, cuando el carril no esté bien fijado a la platabanda alta. Siendo así, en la expresión de la característica de rigidez $s$ se introducirá $J_{t c}=0$. 
Las funciones $H_{i}(z, \mu)$ se expresan como sigue:

$$
\left.\begin{array}{l}
H_{1}(z, \mu)=\frac{(z \cdot \operatorname{ch} z-2 \cdot \operatorname{sh} z) \cdot \operatorname{sh} \mu z-\mu \cdot z \cdot \operatorname{sh} z \cdot \operatorname{ch} \mu z}{\operatorname{sh} 2 z-2 \cdot z} ; \\
H_{2}(z, \mu)=\frac{(z \cdot \operatorname{sh} z-2 \cdot \operatorname{ch} z) \cdot \operatorname{ch} \mu z-\mu \cdot z \cdot \operatorname{ch} z \cdot \operatorname{sh} \mu z}{\operatorname{sh} 2 z+2 \cdot z} ; \\
H_{3}(z, \mu)=\frac{z \cdot \operatorname{ch} z \cdot \operatorname{sh} \mu z-\mu \cdot z \cdot \operatorname{sh} z \cdot \operatorname{ch} \mu z}{\operatorname{sh} 2 z-2 \cdot z} ; \\
H_{4}(z, \mu)=\frac{z \cdot \operatorname{sh} z \cdot \operatorname{ch} \mu z-\mu \cdot z \cdot \operatorname{ch} z \cdot \operatorname{sh} \mu z}{\operatorname{sh} 2 z+2 \cdot z} ; \\
H_{5}(z, \mu)=\frac{(z \cdot \operatorname{ch} z-\operatorname{sh} z) \cdot \operatorname{ch} \mu z-\mu \cdot z \cdot \operatorname{sh} z \cdot \operatorname{sh} \mu z}{\operatorname{sh} 2 z-2 \cdot z} ; \\
H_{6}(z, \mu)=\frac{(z \cdot \operatorname{sh} z-\operatorname{ch} z) \cdot \operatorname{sh} \mu z-\mu \cdot z \cdot \operatorname{ch} z \cdot \operatorname{ch} \mu z}{\operatorname{sh} 2 z+2 \cdot z}
\end{array}\right]
$$

La tabla 1 recoge algunos valores de las funciones adimensionales [1], calculados para algunos puntos $(\zeta, \mu)$ de la placa y los siguientes valores del parámetro $\eta=2,3,5$ y 10 . Como consecuencia del carácter exponencial de las funciones $H_{i}(z, \mu)$, éstas se amortiguan rápidamente con el crecimiento de $z$, diferenciándose poco de cero para valores de la variable $z$ del orden de 7 a 10.

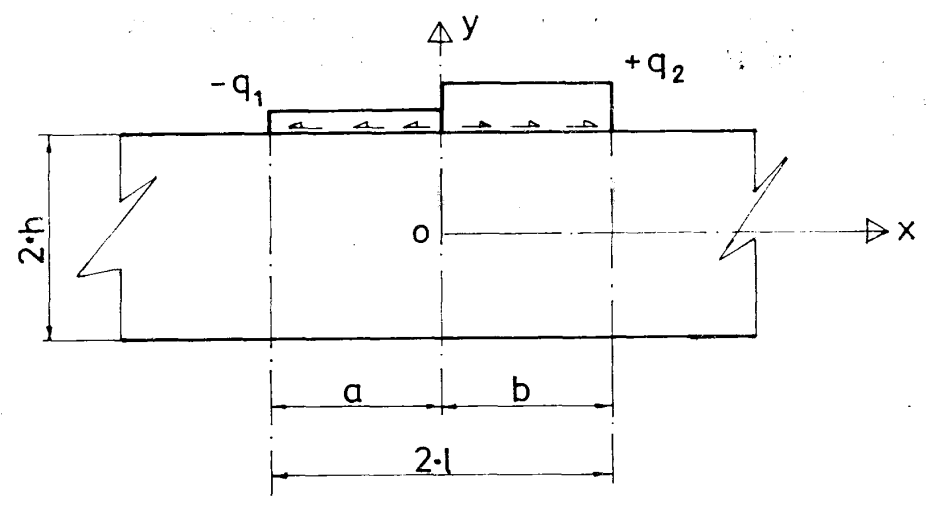

En las figuras 2 y 3, como ejemplo, presentamos los diagramas de las componentes del estado tensional para uno de los valores del parámetro $\eta$.

Del análisis de la solución exacta (tabulada) del sistema [1], Lampsi hace las siguientes deducciones, que conducen a expresiones más simples, y que permiten determinar, con suficiente aproximación, las tensiones en la placa, en casos de carga diferentes del expuesto:

1) De la comparación de los valores obtenidos de las funciones adimensionales $\alpha(\zeta, \eta)$, según la tabla 1, con las que se deducen de la teoría general de la Resistencia de Materiales, como son

$$
\alpha^{0}=\frac{t}{q} \cdot \sigma_{x}^{0}=\frac{t}{q} \cdot\left(\frac{N}{A}+\frac{M}{J} \cdot y\right)
$$

siendo $N=q \cdot(\eta-\zeta) \cdot h$ la fuerza normal,

y $\quad M=q \cdot(\eta-\zeta) \cdot h^{2}$ el momento flector, 
TABLA 1

\begin{tabular}{|c|c|c|c|c|c|c|c|}
\hline$\eta$ & & $\zeta$ & 1,0 & 0,5 & 0,0 & $-0,5$ & $-1,0$ \\
\hline \multirow{3}{*}{2} & $\alpha_{3}^{\prime}$ & $\begin{array}{l}0,0 \\
0,125 \\
0,25 \\
0,5 \\
0,75 \\
1,0 \\
1,5\end{array}$ & $\begin{array}{l}1,5252 \\
1,52062 \\
1,50694 \\
1,4536 \\
1,36936 \\
1,26058 \\
1,00046\end{array}$ & $\begin{array}{l}1,96096 \\
1,95542 \\
1,93126 \\
1,78092 \\
1,52618 \\
1,22924 \\
0,6408\end{array}$ & $\begin{array}{l}0,60704 \\
0,60662 \\
0,6045 \\
0,5874 \\
0,54368 \\
0,47582 \\
0,31914\end{array}$ & $\begin{array}{l}-0,3858 \\
-0,38276 \\
-0,37384 \\
-0,34102 \\
-0,2943 \\
-0,24064 \\
-0,13228\end{array}$ & $\begin{array}{l}-1,5066 \\
-1,49662 \\
-1,46708 \\
-1,35471 \\
-0,18594 \\
-0,98238 \\
-0,55794\end{array}$ \\
\hline & $\beta_{3}$ & $\begin{array}{l}0,0 \\
0,125 \\
0,25 \\
0,5 \\
0,75 \\
1,0 \\
1,5\end{array}$ & $\begin{array}{l}- \\
- \\
- \\
- \\
- \\
-\end{array}$ & $\begin{array}{l}0,54234 \\
0,50548 \\
0,416 \\
0,22626 \\
0,10306 \\
0,0281 \\
-1,0502\end{array}$ & $\begin{array}{c}0,33388 \\
0,32492 \\
0,2997 \\
0,21832 \\
0,12642 \\
0,04346 \\
-0,0931\end{array}$ & $\begin{array}{c}0,1137 \\
0,1114 \\
0,1047 \\
0,08108 \\
0,05028 \\
0,01888 \\
-0,0331\end{array}$ & $\begin{array}{l}- \\
- \\
- \\
- \\
- \\
-\end{array}$ \\
\hline & $\gamma_{3}$ & $\begin{array}{l}0,0 \\
0,125 \\
0,25 \\
0,5 \\
0,75 \\
1,0 \\
1,5\end{array}$ & $\begin{array}{l}\sim \\
1,0 \\
1,0 \\
1,0 \\
1,0 \\
1,0 \\
1,0\end{array}$ & $\begin{array}{c}- \\
-0,03594 \\
-0,0141 \\
0,01412 \\
0,08228 \\
0,11844 \\
0,09728\end{array}$ & \begin{tabular}{l}
\multicolumn{1}{c}{-} \\
$-0,0569$ \\
$-0,10874$ \\
$-0,18522$ \\
$-0,2247$ \\
$-0,23866$ \\
$-0,21756$
\end{tabular} & $\begin{array}{c}- \\
-0,04744 \\
-0,09286 \\
-0,17088 \\
-0,22502 \\
-0,25298 \\
-0,23846\end{array}$ & $\begin{array}{l}- \\
- \\
- \\
- \\
- \\
-\end{array}$ \\
\hline \multirow{3}{*}{3} & $\alpha_{3}$ & $\begin{array}{l}0,0 \\
0,125 \\
0,25 \\
0,5 \\
0,75 \\
1,0 \\
1,5\end{array}$ & $\begin{array}{l}2,95208 \\
2,95412 \\
2,9280 \\
2,85708 \\
2,7423 \\
2,59156 \\
2,20636\end{array}$ & $\begin{array}{l}3,1832 \\
3,1781 \\
3,15404 \\
3,00436 \\
2,7501 \\
2,45176 \\
1,82514\end{array}$ & $\begin{array}{l}1,10006 \\
1,09948 \\
1,0969 \\
1,0774 \\
1,02778 \\
0,94646 \\
0,73082\end{array}$ & $\begin{array}{l}-0,62866 \\
-0,62558 \\
-0,61658 \\
-0,58334 \\
-0,53584 \\
-0,48076 \\
-0,3627\end{array}$ & $\begin{array}{l}-2,49342 \\
-2,49546 \\
-2,45392 \\
-2,34096 \\
-2,16894 \\
-1,9557 \\
-1,46778\end{array}$ \\
\hline & $\beta_{3}$ & $\begin{array}{l}0,0 \\
0,125 \\
0,25 \\
0,5 \\
0,75 \\
1,0 \\
1,5\end{array}$ & $\begin{array}{l}- \\
- \\
- \\
- \\
-\end{array}$ & $\begin{array}{l}0,54308 \\
0,50664 \\
0,41796 \\
0,2315 \\
0,11568 \\
0,05556 \\
0,00518\end{array}$ & $\begin{array}{l}0,33654 \\
0,32804 \\
0,3042 \\
0,22884 \\
0,14864 \\
0,08508 \\
0,0103\end{array}$ & $\begin{array}{l}0,11478 \\
0,11268 \\
0,10666 \\
0,0859 \\
0,0604 \\
0,03696 \\
0,00472\end{array}$ & $\begin{array}{l}- \\
- \\
- \\
- \\
- \\
-\end{array}$ \\
\hline & $\gamma_{3}$ & $\begin{array}{l}0,0 \\
0,125 \\
0,25 \\
0,5 \\
0,75 \\
1,0 \\
1,5\end{array}$ & $\begin{array}{l}- \\
1,0 \\
1,0 \\
1,0 \\
1,0 \\
1,0 \\
1,0\end{array}$ & $\begin{array}{c}- \\
-0,03522 \\
-0,04276 \\
0,01863 \\
0,09322 \\
0,14292 \\
0,17966\end{array}$ & $\begin{array}{l}- \\
-0,05668 \\
-0,10858 \\
-0,1853 \\
-0,2256 \\
-0,24252 \\
-0,24988\end{array}$ & $\begin{array}{l}- \\
-0,04806 \\
-0,09424 \\
-0,17507 \\
-0,23512 \\
-0,2742 \\
-0,30534\end{array}$ & $\begin{array}{l}- \\
- \\
- \\
- \\
- \\
-\end{array}$ \\
\hline
\end{tabular}


TABLA 1 (Cont.)

\begin{tabular}{|c|c|c|c|c|c|c|c|}
\hline$\eta$ & & & 1,0 & 0,5 & 0,0 & $-0,5$ & $-1,0$ \\
\hline \multirow{3}{*}{5} & $\alpha_{3}$ & $\begin{array}{l}0,0 \\
0,125 \\
0,25 \\
0,5 \\
0,75 \\
1,0 \\
1,5\end{array}$ & $\begin{array}{l}6,15238 \\
6,1536 \\
6,1252 \\
6,04176 \\
5,91416 \\
5,73818 \\
5,27392\end{array}$ & $\begin{array}{l}5,59012 \\
5,5831 \\
5,56108 \\
5,41148 \\
5,15746 \\
4,85962 \\
4,23458\end{array}$ & $\begin{array}{l}2,0638 \\
2,06326 \\
2,06078 \\
2,0417 \\
1,99272 \\
1,91302 \\
1,69516\end{array}$ & $\begin{array}{l}-1,11014 \\
-1,10704 \\
-1,08802 \\
-1,06468 \\
-1,01698 \\
-0,96158 \\
-0,8423\end{array}$ & $\begin{array}{l}-4,42032 \\
-4,4104 \\
-4,38106 \\
-4,26892 \\
-4,0983 \\
-3,88702 \\
-3,40292\end{array}$ \\
\hline & $\beta_{3}$ & $\begin{array}{l}0,0 \\
0,125 \\
0,25 \\
0,5 \\
0,75 \\
1,0 \\
1,5\end{array}$ & $\begin{array}{l}- \\
- \\
- \\
- \\
- \\
-\end{array}$ & $\begin{array}{l}0,5425 \\
0,50598 \\
0,41728 \\
0,2309 \\
0,11522 \\
0,05564 \\
0,01042\end{array}$ & $\begin{array}{l}0,3354 \\
0,3269 \\
0,30308 \\
0,22782 \\
0,14806 \\
0,08584 \\
0,0208\end{array}$ & $\begin{array}{l}0,11416 \\
0,11206 \\
0,10604 \\
0,0853 \\
0,06 \\
0,0372 \\
0,00956\end{array}$ & $\begin{array}{l}- \\
- \\
- \\
- \\
- \\
-\end{array}$ \\
\hline & $\gamma_{3}$ & $\begin{array}{l}0,0 \\
0,125 \\
0,25 \\
0,5 \\
0,75 \\
1,0 \\
1,5\end{array}$ & $\begin{array}{l}\sim \\
1,0 \\
1,0 \\
1,0 \\
1,0 \\
1,0 \\
1,0\end{array}$ & $\begin{array}{c}- \\
-0,0355 \\
-0,04316 \\
0,01774 \\
0,09212 \\
0,14178 \\
0,18242\end{array}$ & $\begin{array}{c}- \\
-0,05686 \\
-0,10868 \\
-0,18528 \\
-0,22556 \\
-0,24244 \\
-0,24992\end{array}$ & \begin{tabular}{ll} 
& \multicolumn{1}{c}{-} \\
- & 0,04784 \\
- & 0,09382 \\
- & 0,17424 \\
- & 0,234 \\
- & 0,27314 \\
- & 0,30786
\end{tabular} & $\begin{array}{l}- \\
- \\
- \\
- \\
- \\
-\end{array}$ \\
\hline \multirow{3}{*}{10} & $a_{3}$ & $\begin{array}{l}0,0 \\
0,125 \\
0,25 \\
0,5 \\
0,75 \\
1,0 \\
1,5\end{array}$ & $\begin{array}{l}14,57538 \\
14,56828 \\
14,54702 \\
14,46296 \\
14,32626 \\
14,14158 \\
13,65126\end{array}$ & $\begin{array}{l}11,40374 \\
11,39872 \\
11,3747 \\
11,2251 \\
10,97108 \\
10,67324 \\
10,04818\end{array}$ & $\begin{array}{l}4,38896 \\
4,38936 \\
4,38592 \\
4,36684 \\
4,31786 \\
4,23818 \\
4,02044\end{array}$ & $\begin{array}{l}-2,27346 \\
-2,27038 \\
-2,26134 \\
-2,228 \\
-2,18032 \\
-2,1249 \\
-2,00564\end{array}$ & $\begin{array}{l}-9,0721 \\
-9,0622 \\
-9,03284 \\
-8,9207 \\
-8,75006 \\
-8,5388 \\
-8,0548\end{array}$ \\
\hline & $\beta_{3}$ & $\begin{array}{l}0,0 \\
0,125 \\
0,25 \\
0,5 \\
0,75 \\
1,0 \\
1,5 \\
\end{array}$ & $\begin{array}{l}- \\
- \\
- \\
- \\
- \\
-\end{array}$ & $\begin{array}{l}0,5425 \\
0,50598 \\
0,41728 \\
0,23088 \\
0,10522 \\
0,0556 \\
0,01031\end{array}$ & $\begin{array}{l}0,3354 \\
0,3355 \\
0,30308 \\
0,2278 \\
0,14804 \\
0,08578 \\
0,2056\end{array}$ & $\begin{array}{l}0,11416 \\
0,11206 \\
0,10604 \\
0,0853 \\
0,05998 \\
0,03716 \\
0,00942\end{array}$ & $\begin{array}{l}- \\
- \\
- \\
- \\
- \\
- \\
-\end{array}$ \\
\hline & $\gamma_{3}$ & $\begin{array}{l}0,0 \\
0,125 \\
0,25 \\
0,5 \\
0,75 \\
1,0 \\
1,5\end{array}$ & $\begin{array}{l}1,0 \\
1,0 \\
1,0 \\
1,0 \\
1,0 \\
1,0\end{array}$ & $\begin{array}{c}c \\
-0,0355 \\
-0,04316 \\
0,01774 \\
0,0921 \\
0,14176 \\
0,18228\end{array}$ & $\begin{array}{c}- \\
-0,05686 \\
-0,10868 \\
-0,18528 \\
-0,22556 \\
-0,24244 \\
-0,24992\end{array}$ & $\begin{array}{l}\quad- \\
-0,04784 \\
-0,09321 \\
-0,17424 \\
-0,234 \\
-0,2731 \\
-0,30774\end{array}$ & $\begin{array}{l}- \\
- \\
- \\
- \\
- \\
- \\
-\end{array}$ \\
\hline
\end{tabular}




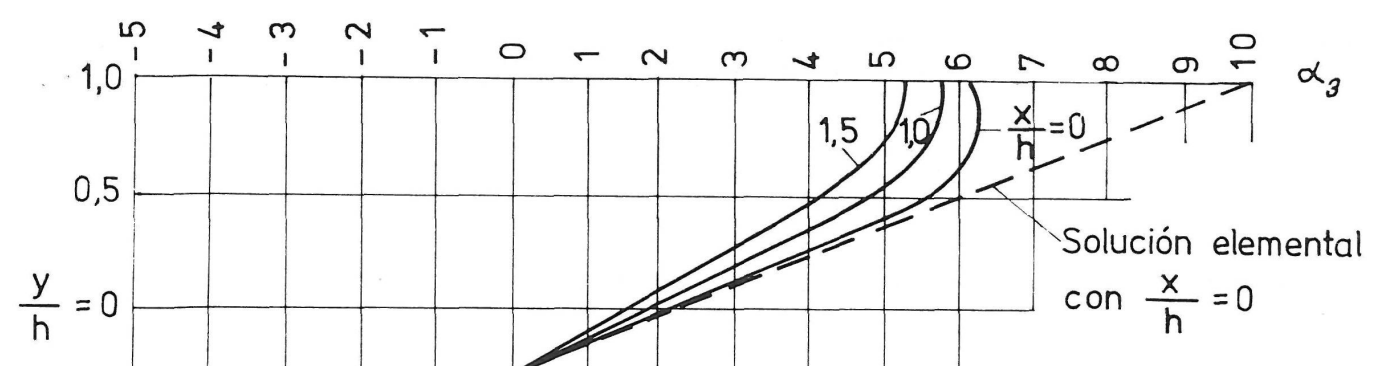

$\alpha_{3}(\xi, \eta)$

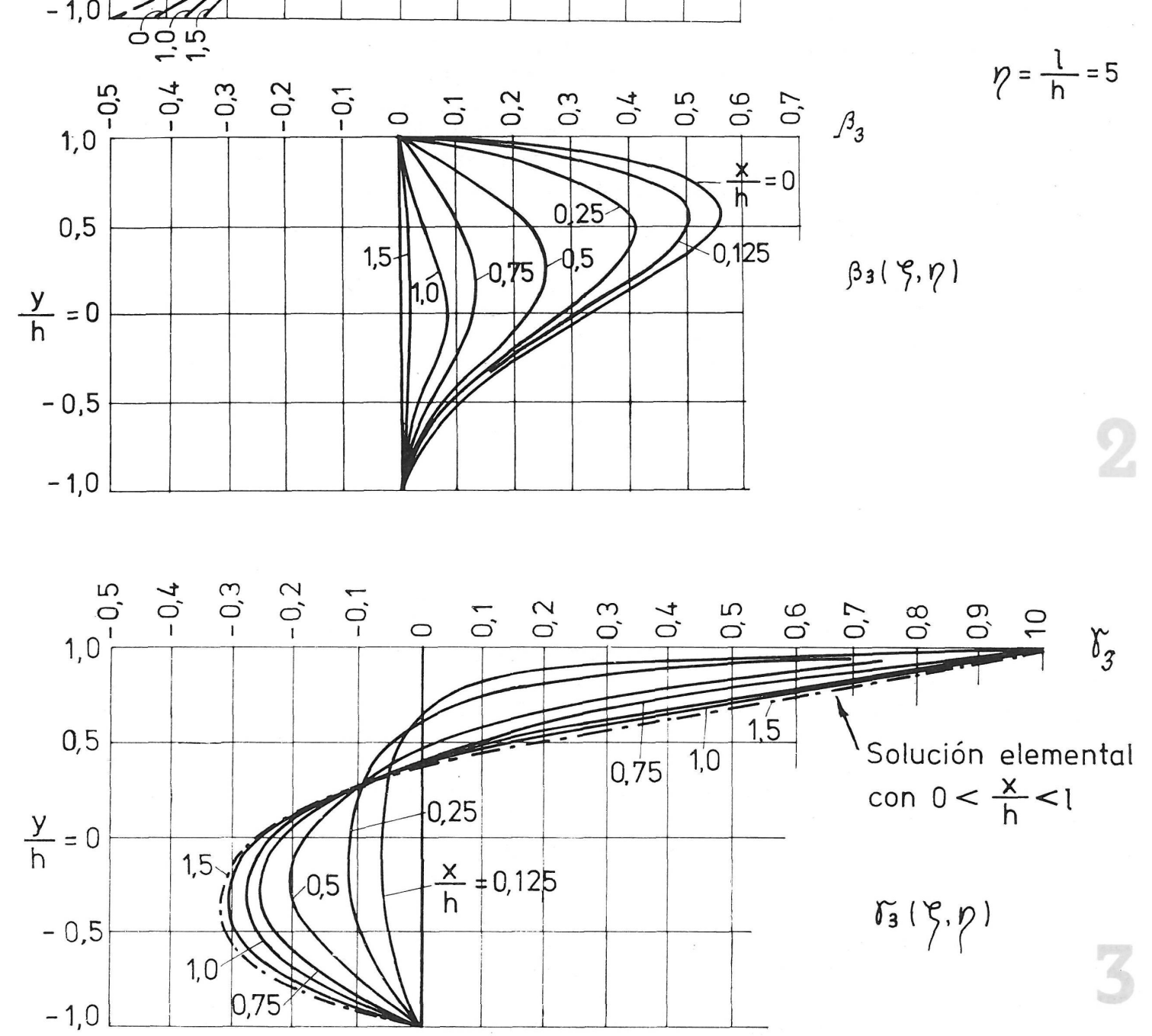

en la sección considerada (para $\zeta \geqslant 0$ ), se desprende que las tensiones locales, determinables por la diferencia

$$
\Delta \alpha=\alpha(\zeta, \eta)-\alpha^{0},
$$

se amortiguan rápidamente al alejarse de la sección en la que se produce la discontinuidad de la intensidad de la carga. Al igual que en el caso de la acción de cargas concentradas $\left(\alpha_{1}, \beta_{1}, \gamma_{1}\right)$, a distancias superiores a la semialtura de la placa, los valores de las tensiones 
locales son suficientemente próximos a cero, como para poderlas despreciar en los cálculos prácticos. A iguales conclusiones se llega en el caso de las funciones adimensionales:

$$
\beta=\frac{t}{q} \cdot \sigma_{y} \quad ; \quad \gamma=\frac{t}{q} \cdot \tau_{x y} ;
$$

siendo para las primeras: $\beta^{0}=0$; y para las segundas:

$$
\gamma^{0}=\frac{t}{q} \cdot \int_{y}^{h} \frac{\partial \sigma_{x}^{0}}{\partial x} \cdot d y .
$$

2) En el entorno $|\zeta|<\eta-1$ de la placa los valores de las tensiones $\sigma_{y}$ y $\tau_{x y}$, prácticamente no dependen de la extensión de la zona sobre la que se encuentra distribuida la carga cortante $q$. Tomando como base lo expuesto en el punto anterior 1), se puede afirmar que en el entorno de $1<|\zeta|<\eta-1$, con $(\eta>2)$, situado a distancias de la sección de discontinuidad de la carga superiores a la semialtura de la placa, la tensión $\sigma_{x}$ depende únicamente de la magnitud de la intensidad de $q$, sin afectarle ni la extensión de la zona sobre la que se halla distribuida la carga, ni la magnitud del salto de la intensidad de la misma en la sección $\zeta=0$.

Es igualmente válido para las tensiones normales $\sigma_{x}$, para un valor cualquiera de $\eta$, superponer, al valor de las mismas obtenido para un $\eta_{1}>|\zeta|+1$, el valor de las tensiones $\sigma_{x}^{0}$, debidas a la carga cortante $q$, distribuida sobre el entorno $\eta_{1} \leqslant \zeta<\eta$, en el borde superior de la placa. Los razonamientos expuestos conducen a las siguientes ecuaciones aproximadas:

$$
\begin{aligned}
& \alpha_{3}(\zeta, \eta)=\alpha_{3}\left(\zeta, \eta_{1}\right)+\frac{K}{2} \cdot(1+3 \cdot \mu) \cdot\left(\eta-\eta_{1}\right) \\
& \beta_{3}(\zeta, \eta)=\beta_{3}\left(\zeta, \eta_{1}\right) \\
& \gamma_{3}(\zeta, \eta)=\gamma_{3}\left(\zeta, \eta_{1}\right)
\end{aligned}
$$

en las que $\eta_{1}<\eta$, y $K$ es un coeficiente corrector de valor igual a 0,78 , en el caso de la fibra $\mu=+1$, e igual a la unidad para las demás. El error que se comete al emplear las expresiones [3] en lugar de la solución exacta (tabla 1), incluso para $\mu=+1$ y con una diferencia entre $\eta_{1}$ y $\eta$ tan grande como 3 ó 10, es inferior al $5 \%$.

Las deducciones expuestas permiten ampliar considerablemente el campo de aplicación de la solución numérica obtenida, extendiéndolo al caso de carga arbitraria asimétrica con respecto al eje $0 y$ de la placa, sin necesidad de realizar, a partir de fórmulas mucho más complicadas que las [1], los cálculos de los correspondientes coeficientes $\alpha, \beta, \gamma$.

En la figura 4 presentamos el caso general de tipo de carga arbitraria aplicada al borde superior de la placa. Si la magnitud $b<a$ es suficientemente grande (por lo menos igual a $h$ ), para determinar las tensiones en el entorno de $|\zeta|<(b / h)-1$, podemos descomponer la carga representada en el esquema I, en las cargas representadas en los esquemas II y III. Las tensiones debidas a la carga, según esquema II, las podemos determinar usando la tabla 1, o a partir de las [3]. Por otra parte, la carga del esquema III la transformamos en la del esquema IV, apoyándonos en las conclusiones del punto 1). En el esquema IV el origen del nuevo sistema de coordenadas $0^{\prime} x^{\prime} y^{\prime}$ coincide con el origen de coordenadas de la sección que investigamos, y toda la carga situada a distancias superiores a la semialtura de la placa la sustituimos por un sistema de fuerzas (fuerzas normales y momentos flectores) estáticamente equivalente a la misma, aplicado en el infinito, puesto que no induce tensiones locales en la sección mencionada. Las tensiones debidas a este último sistema se calculan por la teoría elemental de la Resistencia de Materiales. Lo mismo ocurre con las tensiones $\sigma_{x}$ y $\sigma_{y}$ de la sección $\zeta^{\prime}=0$, debidas a la carga situada en el entorno $-1 \leqslant \zeta^{\prime} \leqslant 1$. 


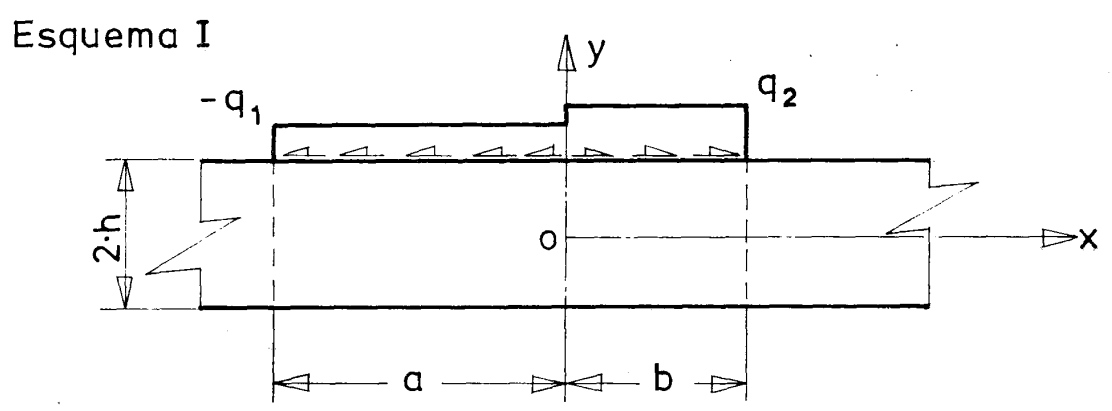

\section{Esquema II}
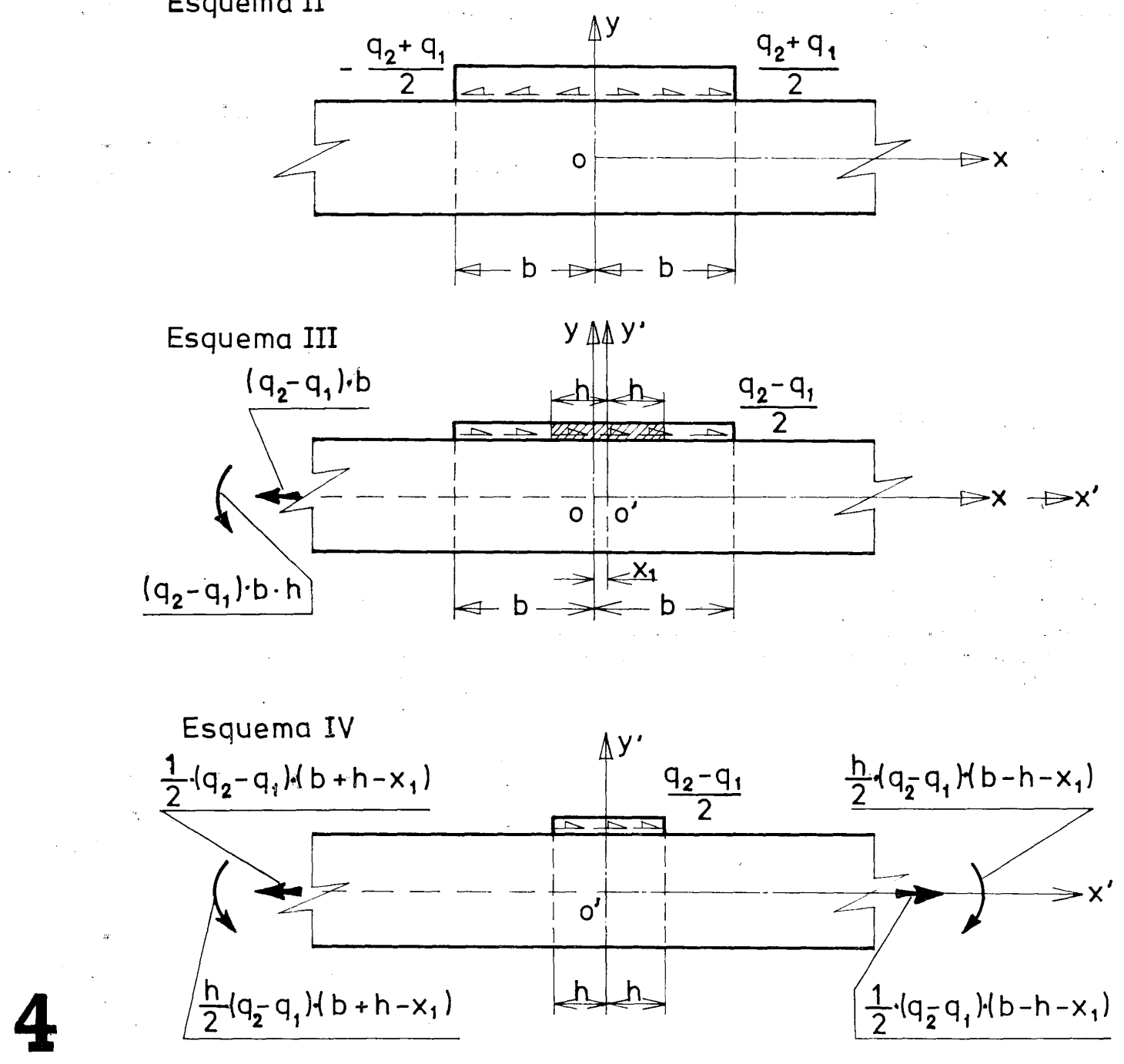

Lampsi, igualmente, llega a la conclusión que para los entornos de $-a+h<x<-h$ y $h<x<b-h$, en el caso de carga según esquema I (fig. 4), las tensiones $\sigma_{x}$, en cualquier sección $x=x_{1}$, se pueden determinar por las fórmulas elementales de la Resistencia de Materiales, como producidas por la carga distribuida a un lado de la sección $x=x_{1}$. Por otra parte, las tensiones $\sigma_{y}$ y $\tau_{x y}$ en los mismos entornos serán constantes. Lo expuesto, junto 
con las expresiones [3], conduce a otro sistema de funciones aproximadas, válidas, por ejemplo, para los entornos $h<x<b-h$, o sea, para $1<\zeta<\eta-1$ :

$$
\begin{aligned}
& \alpha_{3}(\zeta, \eta)=\frac{K}{2} \cdot(\eta-\zeta) \cdot(1-3 \cdot \mu) ; \\
& \beta_{3}(\zeta, \eta)=\beta_{3}(1, \eta) \\
& \gamma_{3}(\zeta, \eta)=\gamma_{3}(1, \eta)
\end{aligned}
$$

Podemos anotar funciones análogas, igualmente, para el entorno de $-a+h<x<-h$. Estas relaciones nos permiten calcular las funciones adimensionales de influencia de las tensiones para aquellos valores de $\zeta$ que se salen de la tabla 1; valores que, en general, no son calculables por las expresiones [3].

Las expresiones y la tabla citadas en este apartado, que recogen el resultado de la investigación realizada por Lampsi, nos permiten calcular, usando un procedimiento sencillo, los valores de las tensiones en las secciones de una placa (el alma) solicitada por cargas tangenciales (cortantes) distribuidas en uno de sus bordes longitudinales.

\section{LAS TENSIONES LOCALES EN LAS VIGAS-CARRILES DE SECCION EN CAJON}

\section{Tensiones de flexión simple de la platabanda alta}

Las soluciones constructivas que elevan la resistencia de las vigas-carriles, de sección en doble te, frente a la fatiga del material producida por los efectos locales orginados por las cargas móviles (soluciones que hemos expuesto en las figuras $17 \mathrm{a}, \mathrm{b}, \mathrm{c}$, de nuestro artículo anterior ${ }^{1}$ ), fueron concebidas e introducidas en la práctica del proyecto de las mismas en los últimos 5 ó 10 años.

En una época anterior los proyectistas norteamericanos, al iniciar la construcción de sus grandes complejos siderúrgicos, que estaban servidos por puentes-grúa de considerable capacidad de elevación, resolvieron el problema prescindiendo de la viga de sección en doble te, e introduciendo la viga-carril de sección en cajón estrécho, con el carril centrado en el plano vertical de simetría de la viga.

Este elemento estructural, llamado por algunos cajón americano, frente a la viga de cajón ancho, o europeo, se hizo muy frecuente en los puentes-grúa, en los puentes de transbordo, etc., y también encontró aplicación, en los últimos años en Europa, como viga-carril en naves industriales, con pasos de más de $18 \mathrm{~m}$ entre soportes, principalmente en la industria siderúrgica ${ }^{10}$.

En la viga-carril de cajón estrecho, en la que el carril es soportado por, y fijado a la platabanda alta, no se considera esta última como apoyo de aquél. Esto obliga a emplear diafragmas cortos con separación de $1 / 3$ a $1 / 4$ del canto de la viga, junto con los diafragmas principales, los cuales son necesarios para garantizar la estabilidad de las almas, y la geometría de la sección del-cajón (fig. 6). Al fijar la separación entre diafragmas cortos, se parte de la idea de que el carril resiste por sí mismo la totalidad del momento flector y esfuerzo cortante producidos por las ruedas de la máquina. Se considera que el carril funciona como viga continua, haciendo de apoyos de la misma los diafragmas, cortos y largos. Sin embargo, la platabanda alta participa en el trabajo de flexión local entre diafragmas, descar- 
gando en parte al carril. Las tensiones repetitivas locales de flexión que se registran en la platabanda, sumadas a las de la flexión general de la viga, hacen descender la resistencia a fatiga de la estructura, y dan lugar a la aparición de fisuras en la zona de mayor concentración de tensiones, como es la unión de la platabanda con el diafragma. La frecuencia de la aparición de este tipo de daños en las vigas en cajón es mucho menor que en el caso de la viga de sección en doble te; pero el peligro existe, y debemos saber atajarlo.

El fenómeno ha sido estudiado por $\operatorname{Odin}^{3}$, quien nos ha proporcionado expresiones finales sencillas, que han pasado con éxito la prueba de la confrontación con los resultados

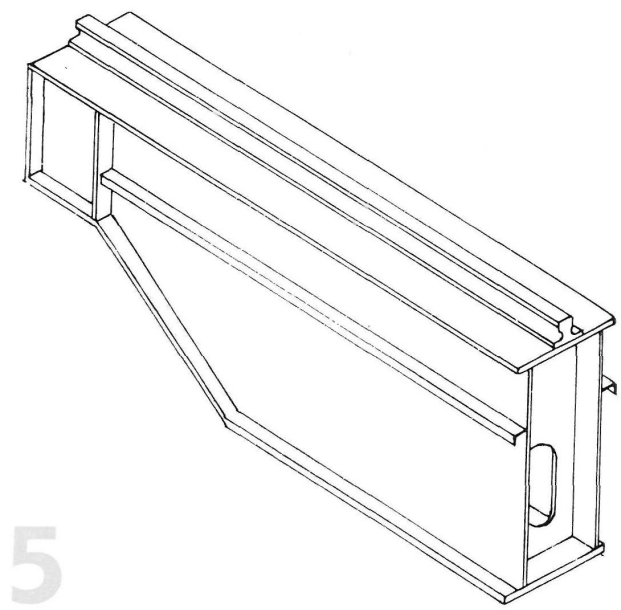
de los ensayos.

Antes de pasar a exponer el razonamiento y las expresiones finales de Odin, procederemos a deducir las expresiones que determinan los corrimientos y los giros de una placa rectangular, que es la parte de platabanda alta, comprendida entre dos diafragmas. Estas expresiones nos serán útiles para seguir el razonamiento de Odin, y las obtendremos, como tantas otras veces, de la obra de Timoshenko, «Teoría de placas y láminas» ${ }^{4}$.

La platabanda alta de una viga en cajón es, desde el punto de vista de la Mecánica, una placa hiperestática apoyada sobre las dos almas de la viga y sobre los diafragmas transversales (fig. 6).

Podemos determinar el ángulo de giro $\varphi_{1}$ en los puntos 1 y 2 de una placa rectangular (fig. 7), solicitada en el centro de su plano por una fuerza concentrada, a partir de la expresión de la flecha, en el punto $y=0$, que según la ${ }^{4}$ es:

$$
\omega_{1}=\frac{a^{2}}{2 \cdot \pi^{3} \cdot D} \cdot \sum_{m=1}^{\infty}\left(\operatorname{th} \alpha_{m}-\frac{\alpha_{m}}{\operatorname{ch}^{2} \alpha_{m}}\right) \cdot \frac{\operatorname{sen} \frac{m \cdot \pi \cdot \xi}{a} \cdot \operatorname{sen} \frac{m \cdot \pi \cdot x}{a}}{m^{3}}
$$

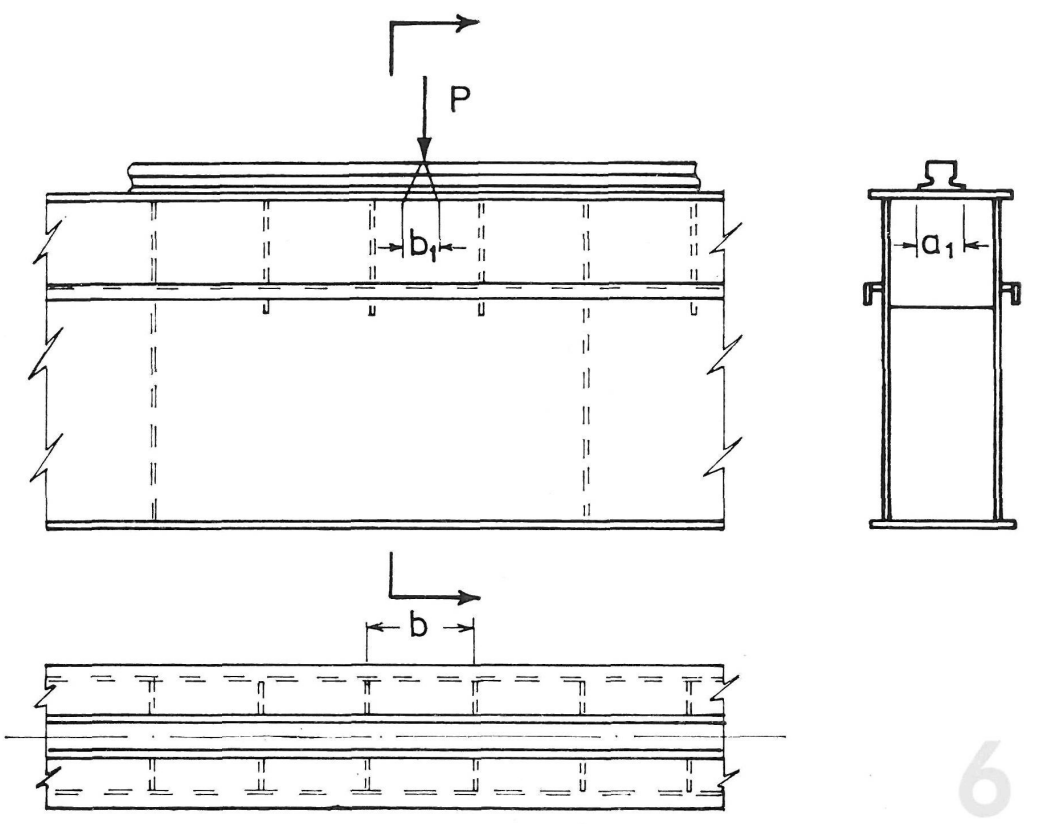



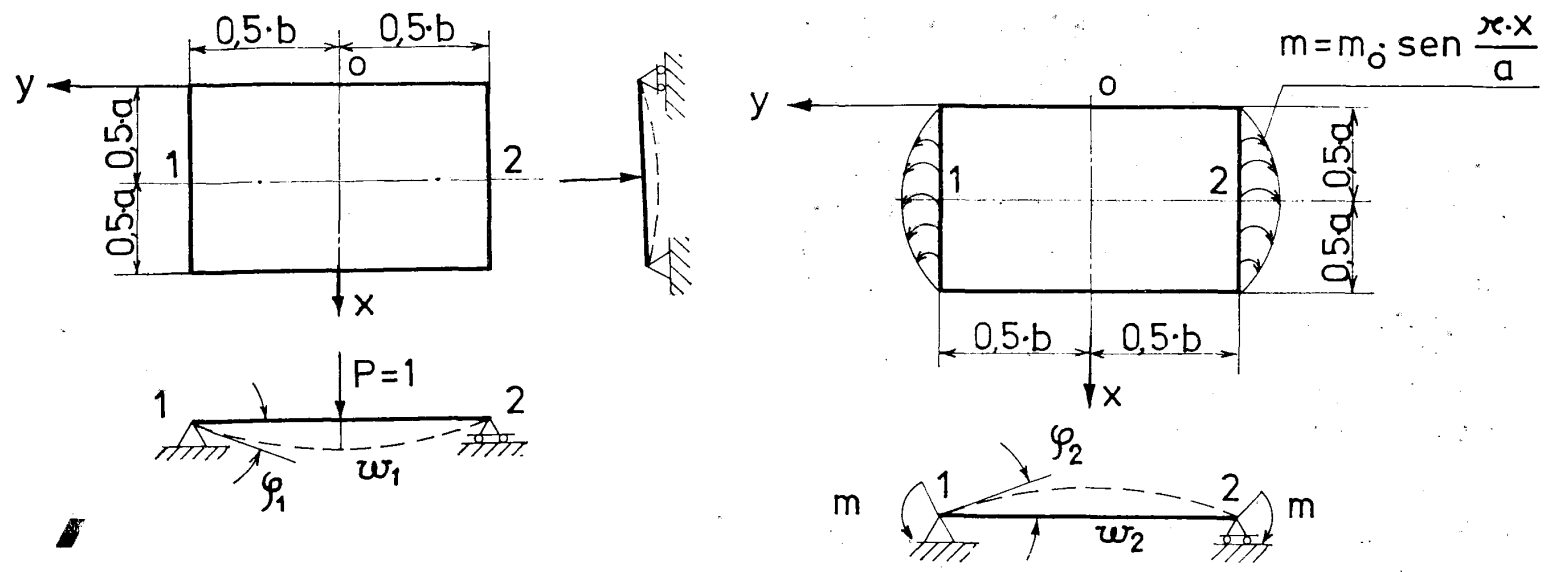

siendo:

$$
\begin{aligned}
\alpha_{m} & =\frac{m \cdot \pi \cdot b}{2 \cdot a} ; \quad \xi=\frac{a}{2} ; \\
D & =\frac{E \cdot t^{3}}{12 \cdot\left(1-\mu^{2}\right)} ;
\end{aligned}
$$

donde:

$\mu=$ Coeficiente de Poisson.

$E=$ Módulo de elasticidad.

$t=$ Espesor de la placa.

Después de diferenciar respecto a $x$, obtenemos para $x=0$ :

$$
p_{1}=\frac{0,56 \cdot \underline{a}}{E \cdot t^{3}} \cdot \sum_{m=1}^{\infty} \frac{1}{m^{2}} \cdot \operatorname{sen} \frac{m \cdot \pi}{2} \cdot\left(\text { th } \alpha_{m}-\frac{\alpha_{m}}{\operatorname{ch}^{2} \alpha_{m}}\right),
$$

que simplificaremos:

$$
\varphi_{1}=\frac{K_{1} \cdot a}{E \cdot t^{3}}
$$

En la tabla 2 figuran los valores del coeficiente $K_{1}$ calculados para algunas relaciones $a: b$.

El ángulo de giro $\varphi_{2}$ en los puntos 1 y 2 de una placa (fig. 8), solicitada en dos lados opuestos por momentos flectores, cuya distribución sigue una ley senoidal, lo podemos determinar partiendo de la expresión de la flecha ${ }^{4}$ :

$$
\begin{gathered}
\omega_{2}=\frac{a^{2}}{2 \cdot \pi^{2} \cdot D} \cdot \sum_{m=1}^{\infty} \frac{\operatorname{sen} \frac{m \cdot \pi \cdot x}{a}}{m^{2} \cdot \frac{\operatorname{ch} \alpha_{m}}{m}} \cdot E_{\mathrm{m}}: \\
\cdot\left(\alpha_{m} \cdot \text { th } \alpha_{m} \cdot \operatorname{ch} \frac{m \cdot \pi \cdot y}{a}-\frac{m \cdot \pi \cdot y}{-a} \cdot \operatorname{sh} \frac{m \cdot \pi \cdot y}{a}\right)
\end{gathered}
$$


TABLA 2. Valores del coeficiente $K_{1}$ en función de la relación $a / b$

\begin{tabular}{|c|c|c|c|c|c|c|c|c|c|c|c|}
\hline$a / b$ & 0,4 & 0,6 & 0,8 & 1,0 & 1,2 & 1,4 & 1,6 & 1,8 & 2,0 & 2,2 & 2,4 \\
\hline$K_{1}$ & 0,0327 & 0,1246 & 0,2325 & 0,3250 & 0,3928 & 0,4383 & 0,4673 & 0,4851 & 0,4959 & 0,5052 & 0,5060 \\
\hline \multicolumn{12}{|c|}{ Valores de los coeficientes $K_{2}$ a $K_{9}$ en función de la relación $b / a$} \\
\hline$b / a$ & 0,4 & 0,6 & 0,8 & 1,0 & 1,2 & 1,4 & 1,6 & 1,8 & 2,0 & 2,2 & 2,4 \\
\hline$K_{2}$ & 1,2209 & 1,5773 & 1,6456 & 1,5930 & 1,5138 & 1,4444 & 1,3938 & 1,3587 & 1,3364 & 1,3226 & 1,3143 \\
\hline$K_{3}$ & 0,3210 & 0,5549 & 0,7710 & 0,9418 & 1,0661 & 1,1517 & 1,2083 & 1,2448 & 1,2674 & 1,2815 & 1,2900 \\
\hline$K_{4}$ & 0,7710 & 1,0661 & 1,2083 & 1,2674 & 1,2900 & 1,2980 & 1,3010 & 1,3018 & 1,3019 & 1,3020 & 1,3021 \\
\hline$K_{5}$ & 0,4500 & 0,5112 & 0,4373 & 0,3256 & 0,2239 & 0,1464 & 0,0928 & 0,0570 & 0,0345 & 0,0206 & 0,0124 \\
\hline$K_{6}$ & 0,1345 & 0,2441 & 0,3047 & 0,3164 & 0,2968 & 0,2622 & 0,2231 & 0,1852 & 0,1511 & 0,1218 & 0,0971 \\
\hline$K_{7}$ & 0,6552 & 0,5636 & 0,4173 & 0,2874 & 0,1907 & 0,1237 & 0,0787 & 0,0492 & 0,0299 & 0,0175 & 0,0097 \\
\hline$K_{8}$ & 0,2744 & 0,4583 & 0,5580 & 0,5689 & 0,5252 & 0,4575 & 0,3845 & 0,3157 & 0,2552 & 0,2040 & 0,1616 \\
\hline$K_{9}$ & 1,9920 & 2,6433 & 2,8539 & 2,8604 & 2,8039 & 2,7424 & 2,6948 & 2,6606 & 2,6383 & 2,6246 & 2,6166 \\
\hline
\end{tabular}

Siendo la carga en forma de momento flector como sigue:

$$
E_{m}=m_{0} \cdot \operatorname{sen} \frac{\pi \cdot x}{a}
$$

y

$$
\alpha_{m}=\frac{m \cdot \pi \cdot b}{2 \cdot a}
$$

hallamos la expresión de $\varphi_{2}$ como la derivada $\frac{d \omega_{2}}{d y}$ para

$$
\begin{aligned}
x & =0,5 \cdot a ; \quad y=0,5 \cdot b \quad \text { y } \quad m_{0}=1 ; \\
\varphi_{2} & =\frac{1,75 \cdot a}{E \cdot t^{3}} \cdot \sum_{m=1}^{\infty} \frac{\operatorname{sen} \frac{m \cdot \pi}{2}}{\mathrm{~m} \cdot \operatorname{ch} \alpha_{m}} \cdot\left[\left(\alpha_{m} \cdot \operatorname{th} \alpha_{m}-1\right) \cdot \operatorname{sh} \alpha_{m}-\alpha_{m} \cdot \operatorname{ch} \alpha_{m}\right] .
\end{aligned}
$$

La expresión obtenida la podemos reducir a una semejante a la [6]:

$$
\varphi_{2}=\frac{K_{2} \cdot a}{E \cdot t^{3}}
$$

La siguiente expresión corresponde a la flecha de una placa solicitada por dos cargas antimétricas de momentos flectores (fig. 9):

$$
\begin{gathered}
\omega_{3}=\frac{a^{2}}{2 \cdot \pi^{2} \cdot D} \cdot \sum_{m=1}^{\infty} \frac{E_{m} \cdot \operatorname{sen} \frac{m \cdot \pi \cdot x}{a}}{m^{2} \cdot \operatorname{sh} \alpha_{m}} \\
\cdot\left(\alpha_{m} \cdot \operatorname{cth} \alpha_{m} \cdot \operatorname{sh} \frac{m \cdot \pi \cdot y}{a}-\frac{m \cdot \pi \cdot y}{a} \cdot \operatorname{ch} \frac{m \cdot \pi \cdot y}{a}\right)
\end{gathered}
$$


Procediendo como en los casos anteriores, obtenemos la expresión del ángulo de giro $\varphi_{3}$ en los puntos 1 y 2 :

$$
\varphi_{3}=\frac{1,75 \cdot a}{E \cdot t^{3}} \cdot \sum_{m=1}^{\infty} \frac{\operatorname{sen} \frac{m \cdot \pi}{2}}{m \cdot \operatorname{sh} \alpha_{m}} \cdot\left[\left(\alpha_{m} \cdot \operatorname{cth} \alpha_{m}-1\right) \cdot \operatorname{ch~} \alpha_{m}-\alpha_{m} \cdot \operatorname{sh} \alpha_{m}\right] .
$$

Después de simplificar, obtenemos para $m=1$ :

$$
\varphi_{3}=\frac{K_{3} \cdot a}{E \cdot t^{3}}
$$
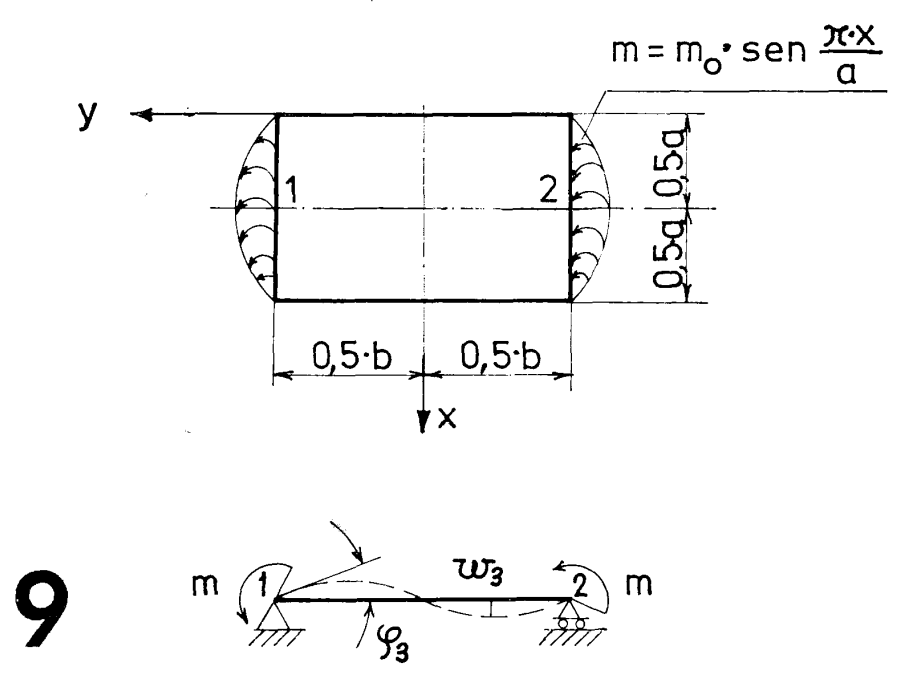

Cuando la placa se halle cargada con momentos flectores

$$
m=\operatorname{sen} \frac{\pi \cdot x}{a}
$$

aplicados en uno solo de sus lados, $y=0,5 \cdot b$, se podrán calcular los valores de los ángulos de giro $\varphi_{4}$, en el punto $1, \mathrm{y} \varphi_{5}$, en el punto 2 , aplicando las siguientes fórmulas:

$$
\varphi_{4}=\frac{K_{4} \cdot a}{E \cdot t^{3}} ; \quad \varphi_{5}=\frac{K_{5} \cdot a}{E \cdot t^{3}}
$$

siendo:

$$
\begin{aligned}
& K_{4}=0,5\left(K_{2}+K_{3}\right) . \\
& K_{5}=0,5\left(K_{2}-K_{3}\right) .
\end{aligned}
$$

La expresión de la flecha máxima en el centro del plano de la placa, cargada, según el esquema de la figura 8 , se puede presentar como sigue:

$$
\omega_{2}=\frac{K_{6} \cdot a^{2}}{E \cdot t^{s}}
$$

en la cual la expresión del coeficiente $K_{6}$ es:

$$
K_{6}=0,56 \cdot \sum_{m=1}^{\infty} \frac{\operatorname{sen} \frac{m \cdot \pi}{2}}{m^{2} \cdot \operatorname{ch} \alpha_{m}} \cdot \alpha_{m} \cdot \text { th } \alpha_{m}
$$


Los momentos flectores $m_{y}$ y $m_{x}$, distribuidos en el centro de la placa, solicitada según el esquema de la figura 8 , se pueden hallar de la expresión general:

$$
\begin{aligned}
& m_{y}=-D \cdot\left(\frac{d^{2} \omega_{2}}{d y^{2}}+\frac{d^{2} \omega_{2}}{d x^{2}}\right) ; \\
& m_{x}=-D \cdot\left(\frac{d^{2} \omega_{2}}{d x^{2}}+\frac{d^{2} \omega_{2}}{d y^{2}}\right) .
\end{aligned}
$$

Las segundas derivadas de la flecha $\omega_{2}$ de la placa (véase la [7]), con $x=0,5 \cdot a$ e $y=0$, tienen por expresión:

$$
\begin{aligned}
& \frac{d^{2} \omega_{2}}{d y^{2}}=\frac{m_{0}}{2 \cdot D} \cdot \sum_{m=1}^{\infty} \operatorname{sen} \frac{m \cdot \pi}{2} \cdot \frac{\alpha_{m} \cdot \operatorname{th} \alpha_{m}-2}{\operatorname{ch} \alpha_{m}} ; \\
& \frac{d^{2} \omega_{2}}{d x^{2}}=-\frac{m_{0}}{2 \cdot D} \cdot \sum_{m=1}^{\infty} \frac{\left(m^{2}+1\right) \cdot \operatorname{sen} \frac{m \cdot \pi}{2} \cdot \alpha_{m} \cdot \operatorname{th} \alpha_{m}}{m^{2} \cdot \operatorname{ch} \alpha_{m}} .
\end{aligned}
$$

Con $\mu=0,3$ las expresiones de los momentos flectores distribuidos correspondientes a las segundas derivadas, son:

A lo largo del eje $y$ :

$$
m_{y}=\frac{m_{0}}{2} \cdot \sum_{m=1}^{\infty} \frac{\operatorname{sen} \frac{m \cdot \pi}{2}}{\operatorname{ch} \alpha_{m}}\left(2-\alpha_{m} \cdot \text { th } \alpha_{m} \frac{0,71 m^{2}-0,3}{m^{2}}\right),
$$

y a lo largo del eje $x$ :

$$
m_{x}=\frac{m_{0}}{2} \cdot \sum_{m=1}^{\infty} \frac{\operatorname{sen} \frac{m \cdot \pi}{2}}{\operatorname{ch} \alpha_{m}}\left(0,6+\alpha_{m} \cdot \operatorname{th} \alpha_{m} \cdot \frac{0,71 m^{2}+1}{m^{2}}\right) .
$$

Ambas expresiones las podemos representar en la siguiente forma simplificada:

$$
\begin{aligned}
& m_{y}=K_{7} \cdot m_{0} \\
& m_{x}=K_{8} \cdot m_{0}
\end{aligned}
$$

La tabla 2 recoge los valores de los coeficientes $K_{2}$ a $K_{8}$.

Después de obtener las expresiones de los corrimientos originados en la placa, por una fuerza concentrada aplicada en el centro de aquélla [6], por cargas simétrica y antimétrica en forma de momentos flectores senoidales aplicados en los apoyos de dos lados [8] y [10], y en los apoyos de un solo lado [11], podemos pasar a exponer la resolución del problema de las tensiones de flexión local de la platabanda alta, según Odin.

Odin considera que la platabanda alta, junto con el carril, forman un sistema mixto, vigaplaca, en la cual la platabanda funciona como placa, y el carril como viga, siendo ambos continuos.

Según Odin, se obtienen resultados, de precisión suficiente, considerando un sistema de sólo tres vanos, generalmente iguales. El contacto entre el carril y la platabanda se localiza en el centro del vano, que es el caso más desfavorable, y se realiza sobre una determinada parte, $b_{1}$, de la separación $b$ entre diafragmas (fig. 6). Este hecho se ha detectado en las 
instalaciones existentes, así como en las series de ensayos llevados a cabo para determinar el valor de $b_{1}$. La existencia de holgurás entre el carril y la platabanda alta es consecuencia tanto de las tolerancias de colocación de los carriles, como de las tolerancias de construcción y montaje de las estructuras metálicas. La investigación estadística ha permitido establecer que la longitud $b_{1}$ se puede fijar igual a la altura de la sección del carril, quedando delimitada la anchura de la huella de contacto por el ancho de la base del carril.

La figura 10 muestra el esquema del sistema estático del que parte Odin, quien considera que la platabanda superior apoya sin empotramiento sobre las almas y los diafragmas del cajón. Esta simplificación se justifica con la gran diferencia de rigideces lineales existente entre los elementos mencionados. Es corriente que el espesor de la platabanda alta sea del orden de 3 a 4 veces mayor que el de las almas o el de los diafragmas.

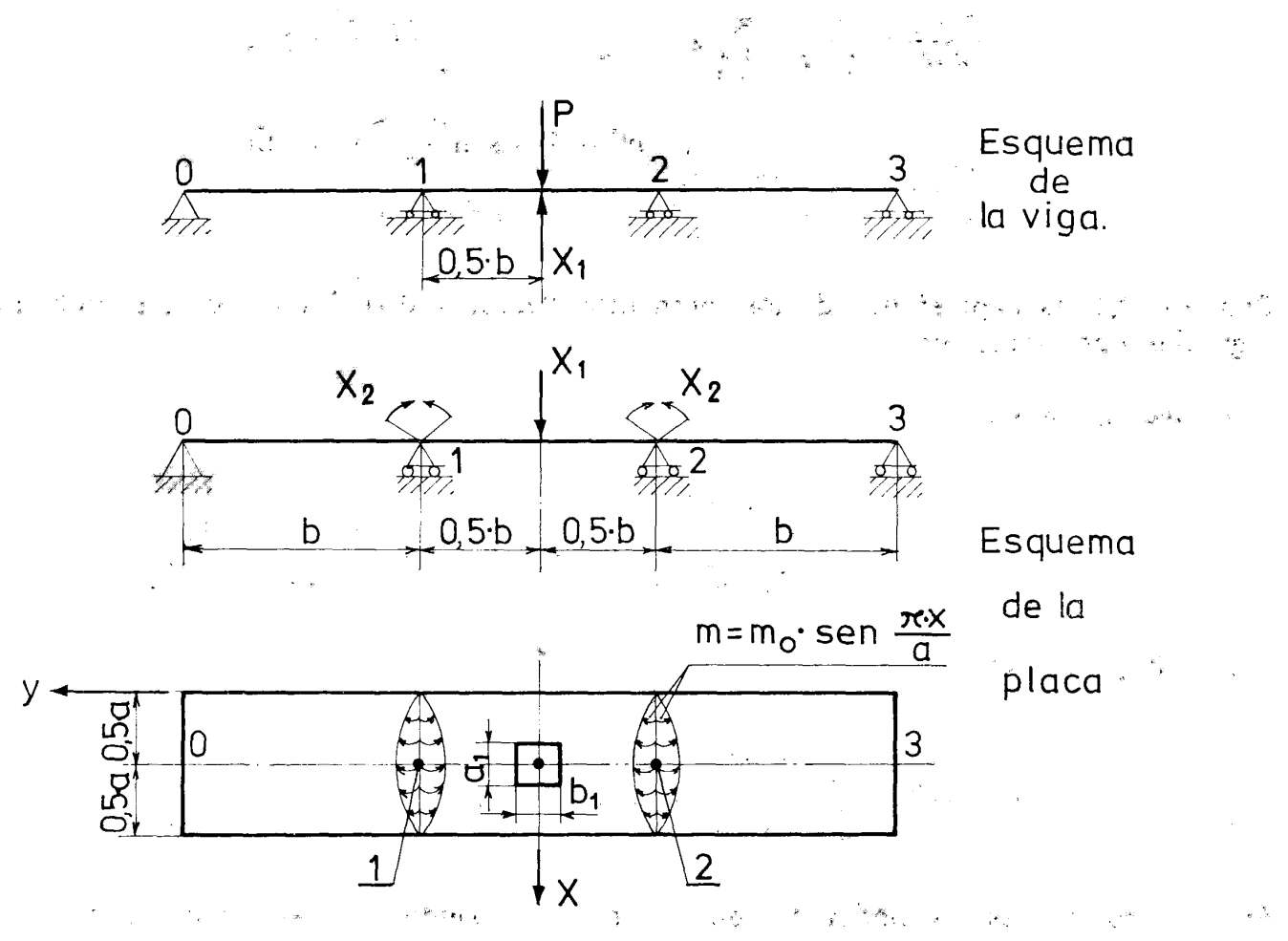

Al determinar las deformaciones de los elementos del sistema presentado, Odin considera que la placa está solicitada en la zona de contacto con el carril por una carga concentrada $X_{1}$, distribuida uniformemente sobre el área $a_{1} \cdot b_{1}$.

Este último supuesto simplifica considerablemente el cálculo a costa de un pequeño error de precisión.

Se pueden considerar los diafragmas como indeformables. El corrimiento relativo de dos diafragmas contiguos, así como el de un diafragma respecto al alma, son magnitudes de segundo orden, en comparación con los corrimientos del carril y de la platabanda alta, que están expuestos a la acción del momento flector local.

En la platabanda, a cada lado de su línea de asiento sobre el diafragma, los momentos flectores $X_{2}$ aparecen distribuidos siguiendo una ley senoidal:

$$
X_{2}=\frac{2}{\pi} \cdot m_{0} \cdot a
$$


El momento flector

$$
m=m_{0} \cdot \operatorname{sen} \frac{\pi \cdot x}{a}
$$

alcanza su valor máximo $m_{0}$ sobre el eje longitudinal de la viga, para descender a cero al acercarse a las almas.

Las incógnitas $X_{1}$ y $X_{2}$ se pueden despejar resolviendo el siguiente sistema de ecuaciones canónicas:

$$
\left.\begin{array}{l}
X_{1} \cdot \delta_{11}+X_{2} \cdot \delta_{12}+\Delta_{1 p}=0 ; \\
X_{1} \cdot \delta_{12}+X_{2} \cdot \delta_{22}=0 .
\end{array}\right]
$$

Al determinar los corrimientos de la viga del sistema mixto, en este caso el carril, no debemos despreciar la influencia del esfuerzo cortante, puesto que la componente correspondiente puede representar casi el $100 \%$ del corrimiento total.

La flecha en el centro del vano central de una viga continua de tres vanos, solicitada por una carga concentrada en el punto en el cual estamos determinando su corrimiento, se compone de la flecha producida por el momento flector, cuya expresión es:

$$
\Delta_{1 p}(M)=-\frac{P \cdot b^{3}}{87 \cdot E \cdot J_{c}}
$$

siendo $J_{c}$ el momento de inercia de la sección del carril, más la flecha debida al esfuerzo cortante:

$$
\Delta_{1 p}(Q)=-\frac{0,652 \cdot K \cdot P \cdot b}{E \cdot A_{c}} ;
$$

donde $A_{c}$ es el área de la sección del carril.

Después de sumar las expresiones, podemos llevar la expresión de la flecha $\Delta_{1 p}$ a la siguiente forma:

$$
\Delta_{\mathrm{tp}}=-\frac{K_{Q} \cdot P \cdot b^{3}}{87 \cdot E \cdot J_{c}}
$$

en la cual, con el coeficiente

$$
K_{Q}=1+\frac{56,5 \cdot K \cdot J_{c}}{A_{c} \cdot b^{2}}
$$

tenemos en consideración la influencia del esfuerzo cortante.

Del curso de Resistencia de Materiales recordamos que la expresión del coeficiente de configuración $K$ es:

$$
K \cdot \frac{A}{J^{2}{ }_{x}} \cdot \int_{z} \frac{S^{2}(z)}{e(z)} \cdot d z
$$

en la cual:

$S(z)=$ Momento estático respecto al eje central, en este caso $x$.

$e(z)=$ Ancho variable de la sección. 
Recordamos que para el rectángulo $K=1,2$; para los carriles de vía férrea el valor de este coeficiente es del orden de 2,7, y para el carril Burbach es del orden de 1,5.

La flecha de la viga, del sistema que comentamos, cargada con $X_{1}=1$ la podemos determinar, entonces, como sigue:

$$
\delta_{11}(v)=\frac{K_{Q} \cdot b^{3}}{87 \cdot J_{c} \cdot E}
$$

y la de la placa del mismo sistema, que de acuerdo con Timoshenko, se obtiene superponiendo a la flecha de una placa simplemente apoyada [5], la flecha producida por los momentos repartidos a lo largo de los dos bordes opuestos [7], es:

$$
\delta_{11}(p)=\frac{K_{0} \cdot c^{2}}{E \cdot t^{3}}
$$

siendo $c$ el menor de los lados de la placa.

El corrimiento total del sistema bajo $X_{1}=1$, en el caso general, viene dado por: ,

$$
\delta_{11}=\frac{1}{E} \cdot\left(\frac{K_{Q} \cdot b^{3}}{87 \cdot J_{c}}+\frac{K_{0} \cdot c^{2}}{t^{3}}\right) .
$$

A continuación presentamos algunos valores del coeficiente $K_{0}$, calculados para siete relaciones del lado más largo, representado por $d$, al más corto, representado por $c$ :

\begin{tabular}{|c|c|c|c|c|c|c|c|}
\hline$d / c$ & 1,0 & 1,1 & 1,2 & 1,4 & 1,6 & 1,8 & 2,0 \\
\hline$K_{0}$ & 0,1265 & 0,1381 & 0,1478 & 0,1621 & 0,1714 & 0,1769 & 0,1803 \\
\hline
\end{tabular}

El ángulo de giro $\delta_{12}$ de la placa en los puntos 1 y 2 , en la intersección de su eje longitudinal con los apoyos, bajo la acción de la fuerza $X_{1}=1$ (véase la [6]), es:

$$
\delta_{12}=\frac{K_{1} \cdot a}{E \cdot t^{3}}
$$

El ángulo total de giro de las placas 0-1 y $1-2$ en los puntos 1 y 2 , debido al momento flector $m=1 \cdot \operatorname{sen}(\pi \cdot x) / a$ aplicado en el apoyo 1 , de ancho $a$, se puede determinar como sigue:

$$
\delta_{22}^{\prime}=\frac{K_{9} \cdot a}{E \cdot t^{3}}
$$

donde $K_{9}=2 \cdot K_{4}+K_{5}$.

Por cuanto, como incógnita $X_{2}=1$ hemos adoptado el momento flector aplicado en todo el largo $a$ del lado 1 , el corrimiento $\delta_{22}$ que le corresponde será $(2 \cdot a) / \pi$ veces menor que $\delta_{22}^{\prime}$, que hemos deducido en el supuesto de acción de un momento flector de magnitud $1 \cdot \operatorname{sen}(\pi \cdot x) /$ a por unidad de longitud.

En consecuencia:

$$
\delta_{22}=\frac{K_{9} \cdot \pi}{2 \cdot E \cdot t^{3}}
$$


Una vez resuelto el sistema [16], despejamos las incógnitas $X_{1}$ y $X_{2}$ :

$$
\left.\begin{array}{l}
X_{1}=K_{9} \cdot A, \\
X_{2}=0,64 \cdot K_{1} \cdot a \cdot A
\end{array}\right]
$$

así como el valor máximo del momento flector en los puntos 1 y 2 :

$$
m_{0}=K_{1} \cdot A .
$$

La tensión correspondiente en la sección de apoyo de la placa, en la dirección longitudinal es:

$$
\sigma_{\text {apoyo }}= \pm \frac{6 \cdot m_{0}}{t^{2}} .
$$

Los coeficientes que hemos dejado sin definir en las expresiones anteriores son:

$$
A=\frac{P \cdot B}{K_{9} \cdot\left(B+87 K_{0} \cdot c^{2}\right)-55 \cdot K_{1}^{2} \cdot a^{2}}
$$

y:

$$
B=\frac{K_{Q} \cdot b^{3} \cdot t^{3}}{J_{c}},
$$

El momento flector que soporta el carril en la mitad del vano entre diafragmas es, entonces:

$$
M_{c}=0,175 \cdot\left(P-X_{1}\right) \cdot b .
$$

Las tensiones en la platabanda alta de la viga cajón, en el centro de la placa, se calculan como sigue:

$$
\sigma_{0}= \pm\left(\sigma_{0 p}+\sigma_{0 m}\right)
$$

en la que:

$\sigma_{0 p}=$ Tensión inducida por la fuerza $X_{1}$.

$\sigma_{0 m}=$ Tensión debida a los momentos flectores $m$, repartidos a lo largo del lado a de la placa.

Por cuanto hemos supuesto que la fuerza $X_{1}$ está aplicada uniformemente sobre el rectángulo $a_{1} \cdot b_{1}$, la tensión $\sigma_{y p}$ en dirección longitudinal será:

$$
\sigma_{y p}=\frac{K_{10} \cdot X_{1}}{t^{2}}
$$

y en dirección normal:

$$
\sigma_{x p}=\frac{K_{11} \cdot X_{1}}{t^{2}}
$$

En las tablas 3 y 4 presentamos algunos valores de los coeficientes respectivos $K_{10}$ y $K_{11}$ para el caso $b>a$. En caso de ser $b<a$ se debe, para mayor facilidad de uso de las tablas, invertir los índices, girando $90^{\circ}$ los ejes de coordenadas. 
TABLA 3. Valores del coeficiente $K_{10}$ en función de las dimensiones de la placa y del área de aplicación de la carga

\begin{tabular}{|c|c|c|c|c|c|c|c|c|c|}
\hline \multirow{3}{*}{$b_{1} / a$} & \multicolumn{3}{|c|}{$b: a=1$} & \multicolumn{3}{|c|}{$b: a=1,4$} & \multicolumn{3}{|c|}{$b: a=1,8$} \\
\hline & \multicolumn{9}{|c|}{$a_{1} / a$} \\
\hline & 0,2 & 0,4 & 0,6 & 0,2 & 0,4 & 0,6 & 0,2 & 0,4 & 0,6 \\
\hline 0,2 & 1,284 & 1,073 & 0,900 & 1,248 & 1,050 & 0,882 & 1,224 & 1,026 & 0,864 \\
\hline 0,4 & 0,966 & 0,846 & 0,726 & 0,942 & 0,828 & 0,714 & 0,924 & 0,804 & 0,690 \\
\hline 0,6 & 0,762 & 0,678 & 0,594 & 0,750 & 0,666 & 0,582 & 0,726 & 0,642 & 0,564 \\
\hline
\end{tabular}

TABLA 4. Valores del coeficiente $K_{11}$ en función de las dimensiones de la placa y del área de aplicación de la carga

\begin{tabular}{|c|c|c|c|c|c|c|c|c|c|}
\hline \multirow{3}{*}{$b_{1} / a$} & \multicolumn{3}{|c|}{$b: a=1$} & \multicolumn{3}{|c|}{$b: a=1,4$} & \multicolumn{3}{|c|}{$b: a=1,8$} \\
\hline & \multicolumn{9}{|c|}{$a_{1} / a$} \\
\hline & 0,2 & 0,4 & 0,6 & 0,2 & 0,4 & 0,6 & 0,2 & 0,4 & 0,6 \\
\hline 0,2 & 1,284 & 0,966 & 0,762 & 1,435 & 1,117 & 0,912 & 1,500 & 1,182 & 0,966 \\
\hline 0,4 & 1,073 & 0,846 & 0,678 & 1,284 & 1,010 & 0,828 & 1,314 & 1,074 & 0,888 \\
\hline 0,6 & 0,900 & 0,726 & 0,594 & 1,087 & 0,906 & 0,756 & 1,152 & 0,966 & 0,810 \\
\hline
\end{tabular}

Sin embargo, se debe retener la verdadera dirección de las tensiones que se estén calculando. Después de introducir en la [19], por una parte, las expresiones [14], [18] y [20] y, por otra, las [15], [18] y [21], obtenemos las expresiones finales:

$$
\left.\begin{array}{l}
\sigma_{0 y}= \pm \frac{A}{t^{2}} \cdot\left(K_{9} \cdot K_{10}-6 \cdot K_{1} \cdot K_{7}\right) ; \\
\sigma_{0 x}= \pm \frac{A}{t^{2}} \cdot\left(K_{9} \cdot K_{11}-6 \cdot K_{1} \cdot K_{8} \cdot\right.
\end{array}\right]
$$

En la figura 11 puede verse el esquema estático correspondiente al caso de la fuerza $P$ situada en el centro del primer vano. Este caso se presenta en los extremos del carril o

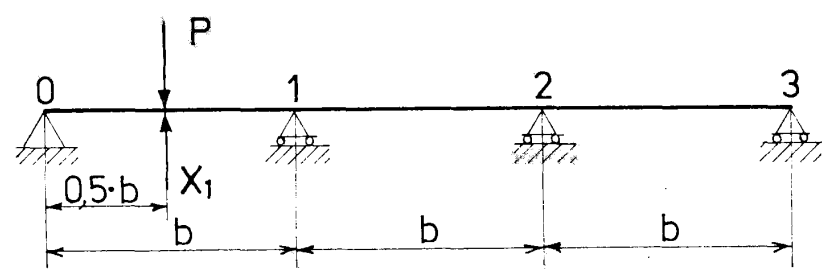


en una junta del mismo. El corrimiento bajo la carga $P$ en dirección de la incógnita $X_{1}$ es, en este caso:

$$
\Delta_{1 p}=-\frac{K^{\prime \prime}{ }_{Q} \cdot P \cdot b^{3}}{68,5 \cdot E \cdot J_{c}} .
$$

El corrimiento debido a $X_{1}$ en la dirección de la misma, así como los coeficientes correspondientes se determinan como sigue:

$$
\begin{aligned}
\delta_{11} & =\frac{1}{E} \cdot\left(\frac{K^{\prime \prime}{ }_{Q} \cdot b^{3}}{68,5 \cdot E \cdot J_{c}}+\frac{K_{0} \cdot c^{2}}{t^{3}}\right) ; \\
K^{11}{ }_{Q} & =1+\frac{47,5 \cdot K \cdot J_{c}}{b^{2} \cdot A_{c}} ; \\
A & =\frac{P \cdot B}{K_{9} \cdot\left(B+68,5 \cdot K_{0} \cdot c^{2}\right)-39,2 \cdot K^{2} \cdot a^{2}} .
\end{aligned}
$$

Las magnitudes restantes, $\operatorname{como} B, X_{1}, X_{2}, m_{0}, \sigma_{a p} \mathrm{y} \sigma_{0}$, se determinan igual que antes.

Son peligrosas, desde el punto de vista de la fatiga del material, las tensiones $\sigma_{\Sigma}$ totales que aparecen en la platabanda alta, en su unión con un diafragma, tensiones que se calculan como sigue:

$$
\sigma_{\Sigma}=\sigma_{f g}+\sigma_{a p}
$$

donde $\sigma_{f g}$ es la tensión de flexión general de la viga-cajón.

Los cálculos realizados, siguiendo a Odin, así como los resultados obtenidos en los ensayos e investigaciones, indican la posibilidad de la aparición de elevadas tensiones de flexión local en las platabandas altas de las vigas-carriles de sección en cajón; tensiones locales éstas que no se deben despreciar en el proyecto de la estructura, sobre todo en naves servidas con puentes-grúa de régimen de servicio pesado e, incluso, mediano.

\section{Tensiones normales secundarias de la flexión coartada}

Otro tipo de fenómeno local de cierta importancia, que debemos tener en cuenta en el proyecto de las vigas de sección en cajón, es el de la flexión coartada.

En la práctica, las vigas en cajón se calculan, generalmente, como vigas de sección maciza, partiendo del supuesto que las deformaciones longitudinales, y las correspondientes tensiones normales, se distribuyen uniformemente en todo el ancho de las platabandas y que las deformaciones longitudinales de las fibras de las almas varían siguiendo una ley lineal (Hipótesis de Bernouilli).

Sin embargo, en la realidad, las vigas de sección en cajón son estructuras que están constituidas por placas de pequeño espesor y tienen una elevada sensibilidad a la influencia de las tensiones tangenciales, lo cual es una particularidad que caracteriza a todo elemento de pequeño espesor frente a los de sección maciza.

En el estudio del funcionamiento de una viga bajo la acción de cargas situadas en su plano vertical de simetría, se presupone que en las secciones transversales de la viga tiene lugar el fenómeno de flexión simple libre, en el cual las fibras longitudinales no ejercen presión unas sobre otras, y no existe coacción alguna para el libre alabeo de las secciones transversales.

Experimentalmente se confirma la ausencia de presiones entre las distintas fibras en la flexión libre, teniendo lugar cierta alteración de la forma plana de las secciones transversales. 
Estas alteraciones son, como se sabe, consecuencia inevitable de los deslizamientos introducidos en la flexión simple libre por los esfuerzos tangenciales.

En la figura 12 puede observarse la forma que toma una porción de la viga en cajón flectada, cuando se cumplen las condiciones de la flexión pura, y en la figura 13 vemos la forma que adquiere la misma porción bajo la influencia de los esfuerzos tangenciales, en condiciones de flexión simple libre. En la primera figura las secciones transversales de la viga permanecen planas, mientras que en la segunda aparecen alabeadas.

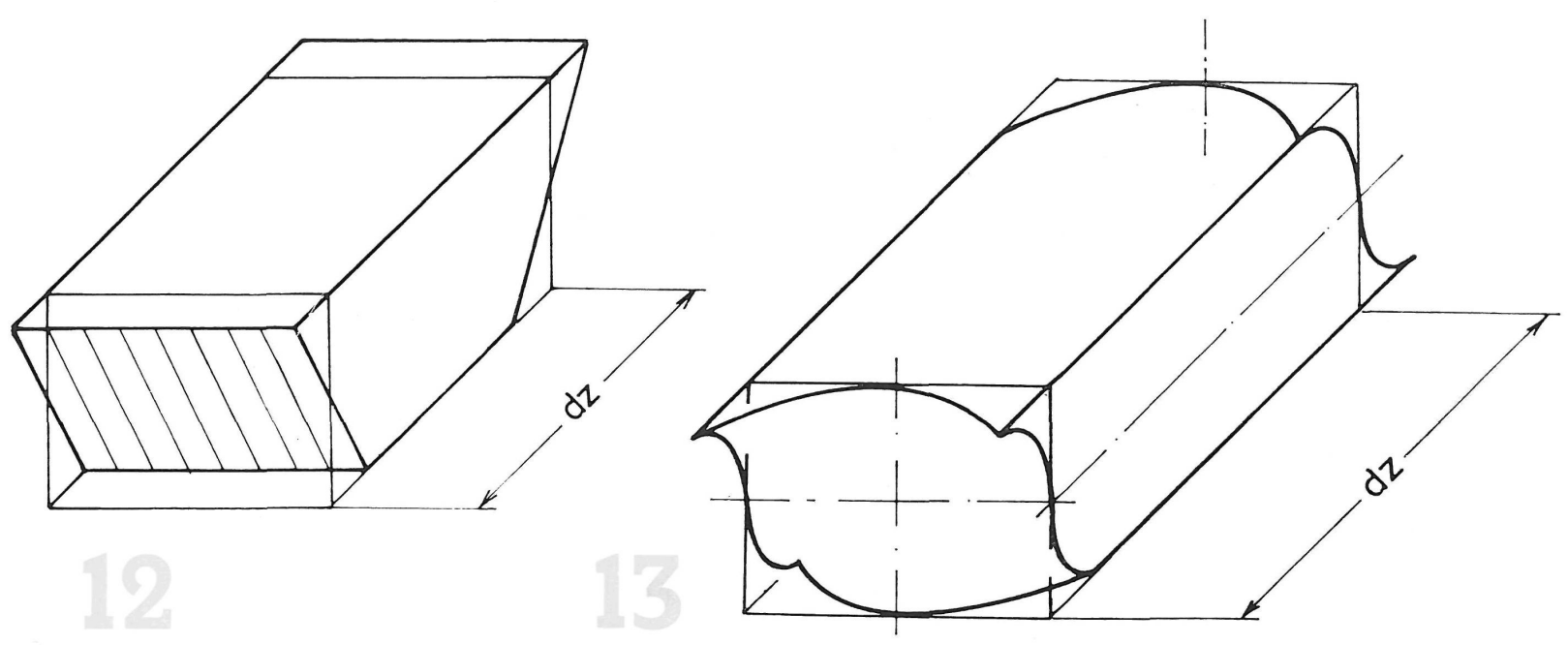

En consecuencia, podemos afirmar que en toda sección de la viga, donde exista alguna coacción, o no exista la posibilidad de realizarse el libre alabeo de las secciones, aparecen tensiones normales secundarias que se oponen al alabeo libre de la sección y tienden a conservarla en su plano inicial.

Como resultado, observamos en los ensayos que las tensiones normales dejan de tener un valor uniforme en todo el ancho de la platabanda, y que en las almas no siguen una ley lineal.

Dado que las tensiones secundarias aparecen como resultado de la coacción del alabeo de las secciones, a este tipo de flexión se suele denominar flexión simple coartada, por analogía con la torsión coartada.

Los fenómenos de flexión coartada aparecen en las secciones empotradas y en su entorno; en la sección central y su entorno, de la viga simple; en el entorno de las secciones rigidizadas por diafragmas; etc.

La flexión coartada fue investigada por S. Kahn ${ }^{5}$, en los últimos años de la década de los 40, en su aplicación a las estructuras monocasco de los aviones.

En la figura 14 hemos representado el diagrama de las tensiones normales totales que aparecen en una sección de la viga en cajón en la flexión coartada. Con línea a trazos representamos el diagrama de las tensiones normales en la flexión libre. La figura 15 muestra el diagrama característico de distribución de las tensiones normales secundarias originadas por la coacción en una platabanda, mientras que la figura 16 representa el diagrama correspondiente a las tensiones normales en un alma.

A continuación presentamos, siguiendo a Parñitsky ${ }^{6,7}$ en su adaptación del método de Kahn al caso más simple de la viga en cajón de un solo vano, algunas expresiones finales que se- 

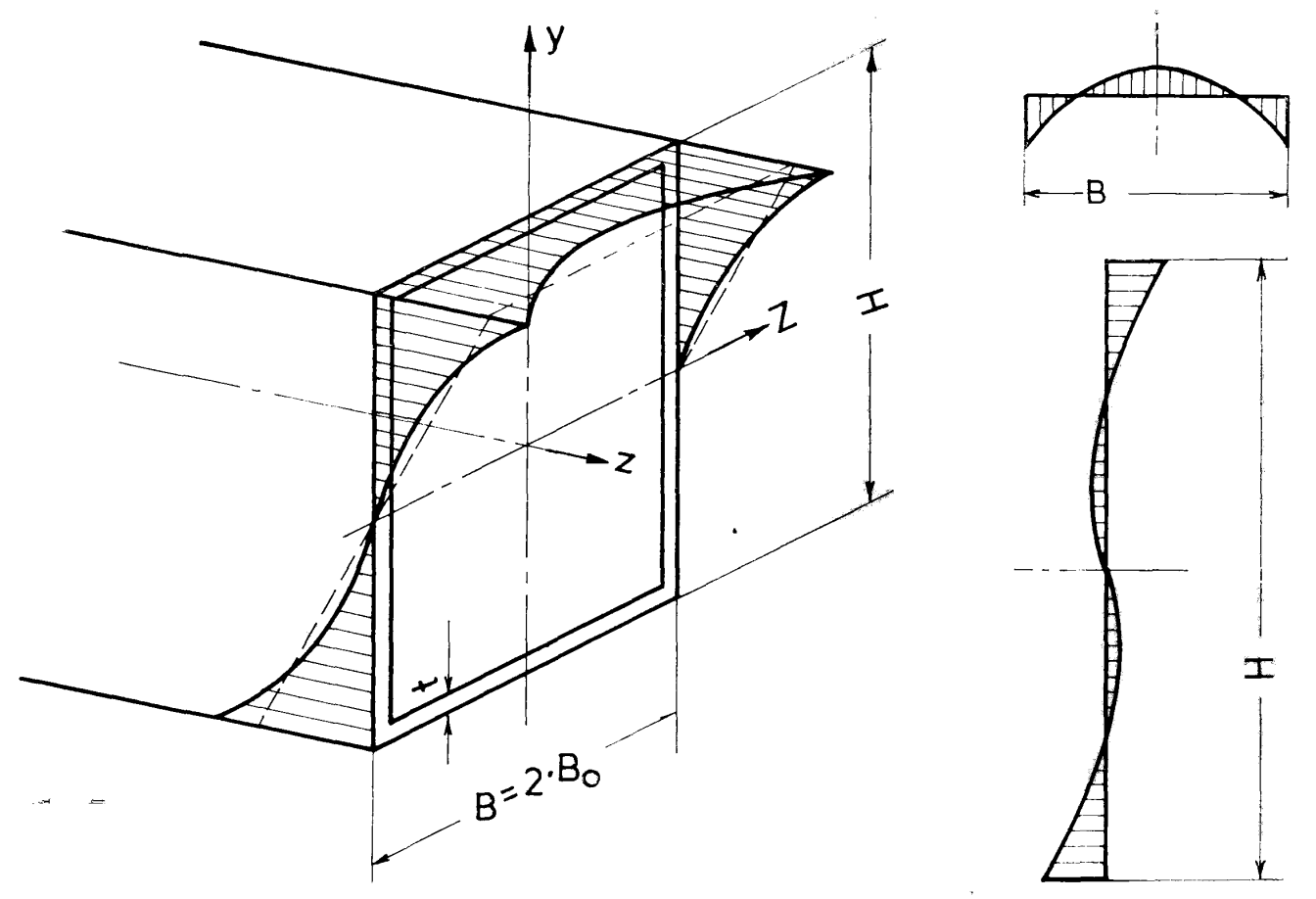

rán suficientes para que el Ingeniero proyectista pueda determinar con rapidez la influencia de las tensiones normales secundarias de coacción de la flexión libre.

Repetimos, que en la viga de un solo vano con apoyo isostático las tensiones secundarias de flexión coartada aparecen en la sección situada en el centro del vano y en las de su entorno, donde las secciones no pueden alabearse con libertad.

Hacemos las siguientes simplificaciones de partida:

- Despreciamos los pequeños vuelos constructivos de la platabanda que la viga en cajón suele llevar, para facilitar el amarre de conductores, por ejemplo.

Consideraremos, por consiguiente, que el ancho de la platabanda es igual a la separación entre las almas de la viga.

- Al determinar la influencia de la coacción sobre la distribución de las tensiones en el ancho de la platabanda, suponemos que las platabandas son de espesor suficiente para absorber tensiones normales y tangenciales, mientras que las almas, más delgadas que las primeras, trabajan sólo a cortadura.

- Suponemos que las tensiones normales secundarias, originadas por la coacción, son proporcionales a las alteraciones de los corrimientos de la flexión libre. Sabemos que las tensiones normales secundarias tienden a eliminar el alabeo de las secciones. Por consiguiente, en aquellos puntos de la sección en los que en la flexión libre tienen lugar grandes corrimientos de alabeo, en la flexión coartada aparecen tensiones normales secundarias elevadas, y, por el contrario, en las secciones en las que en la flexión libre sólo se realizan pequeños corrimientos de alabeo, aparecen, en la flexión coartada, tensiones secundarias de poca importancia.

Siguiendo la teoría de Kahn determinamos las tensiones normales finales en cada sección, como el resultado de la superposición de las tensiones normales de flexión libre, que siguen 
la ley lineal, con las tensiones normales secundarias debidas a la coacción, que siguen la ley de una parábola cuadrática:

$$
\sigma=\frac{M(z)}{J_{x}} \cdot y+\varphi(z) \cdot \omega
$$

teniendo en cuenta que las tensiones normales secundarias son proporcionales a los corrimientos longitudinales $\omega$, debidos al alabeo de las secciones transversales planas, en la dirección del eje $z$ (longitudinal de la viga), y que tiene su origen en el centro de la sección del apoyo.

Al determinar la influencia que en la platabanda ejerce la coacción sobre la flexión libre, consideramos los corrimientos de un prisma elemental, de dimensiones $d s, d z$, $t$, siendo $d s$ el ancho medido en la dirección de la línea $s$ del contorno de la sección transversal (en este caso a lo ancho de la platabanda); $d z$, la longitud del prisma, medida en dirección paralela al eje longitudinal $z$ de la viga; y $t$, la altura del prisma, que es igual al espesor de la platabanda.

Para la viga de sección en cajón de doble simetría la función $\omega$ se puede determinar por la fórmula siguiente:

$$
\bar{\omega}=\int_{0}^{s} \frac{S(x)}{t} \cdot d s+\omega^{\prime} \cdot y ;
$$

en la cual:

$S(x)=$ Momento estático que determina el valor de los esfuerzos tangenciales unitarios de flexión, en el supuesto de que la fuerza exterior (la carga) pase por el centro de torsión de la sección.

$\omega^{\prime}=$ Una magnitud constante que se determina como sigue:

$$
\omega^{\prime}=\frac{\int_{\text {contorno }}\left[\int_{0}^{s} \frac{S(x)}{t} \cdot d s\right] \cdot t \cdot y \cdot d s}{J_{x}} ;
$$

siendo $J_{x}$ el momento de inercia de la sección completa.

En el caso de la platabanda de la viga-cajón de anchura $2 \cdot B_{0}$ la ecuación de $\bar{\omega}$, que representamos gráficamente en la figura 17 , se anota como sigue:

$$
\int_{0}^{s} \frac{S \cdot(x)}{t} d s-\frac{B_{0}{ }^{2} \cdot H}{6}
$$

Con $\bar{\omega}=1$ indicamos el corrimiento máximo en el vértice de la sección de la viga. La ecuación diferencial que determina las tensiones normales en la fibra que sufre un corrimiento $\bar{\omega}=1$, deducida en el supuesto de que la carga exterior se transmita a la viga-cajón en forma continua a través de un número infinito de diafragmas, es:

$$
\frac{d^{2} \sigma}{d z^{2}}-u^{2} \cdot \sigma=-\eta \cdot M(z)+\gamma \cdot \frac{d^{2} M(z)}{d z^{2}}
$$

siendo:

$\frac{d^{2} M(z)}{d z^{2}}=\frac{d Q(z)}{d z^{2}}=q=$ Carga que soporta la viga por unidad de longitud. 


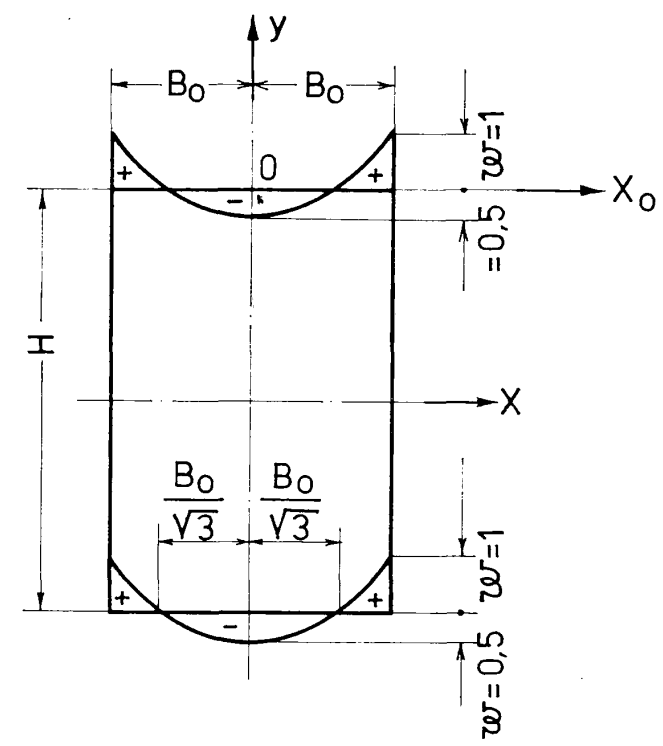

$u, \eta, \gamma=$ Coeficientes, que para la viga de sección en cajón (fig. 14) tienen los siguientes valores:

$$
u=\frac{2}{B_{0}} \quad ; \quad \eta=\frac{2}{B_{0}^{2} \cdot H \cdot t} \quad ; \quad \gamma=\frac{2,25}{B_{0} \cdot H \cdot t} .
$$

Podemos anotar la solución de la [25] como sigue:

$$
\sigma=D_{1} \cdot \operatorname{sh} u z+D_{2} \cdot \operatorname{ch} u z+\sigma_{r} ;
$$

en la que:

$D_{1}$ y $D_{2}=$ Constantes que se determinan a partir de las condiciones en el entorno, así:

- en el extremo libre: $\sigma=0$;

- en el empotramiento: $\frac{d \sigma}{d z}=\gamma \cdot \frac{d M(z)}{d z}$;

$\sigma_{r}=$ Solución particular, dependiente tanto de la ley de variación, de los momentos flectores $M(z)$, a lo largo de la viga, como de la de carga repartida $q$.

Centrémonos en el caso de la viga de un solo vano, con apoyo isostático, que soporta una carga concentrada $P$ en el centro del vano, de longitud $L$ (fig. 18). Como la sección central de la viga permanece plana, son aplicables las condiciones de un empotramiento. En este caso:

$$
M_{(0,5 \cdot L)}=0,5 \cdot P \cdot z .
$$

De la ecuación diferencial [25] obtenemos que:

$$
\sigma_{r}=0,5 \cdot P \cdot \frac{\eta}{u^{2}} \cdot z,
$$

y por consiguiente:

$$
\sigma=D_{1} \cdot \operatorname{sh} u z+D_{2} \cdot \operatorname{ch} u z+0,5 \cdot P \cdot \frac{\eta}{u^{2}} \cdot z
$$




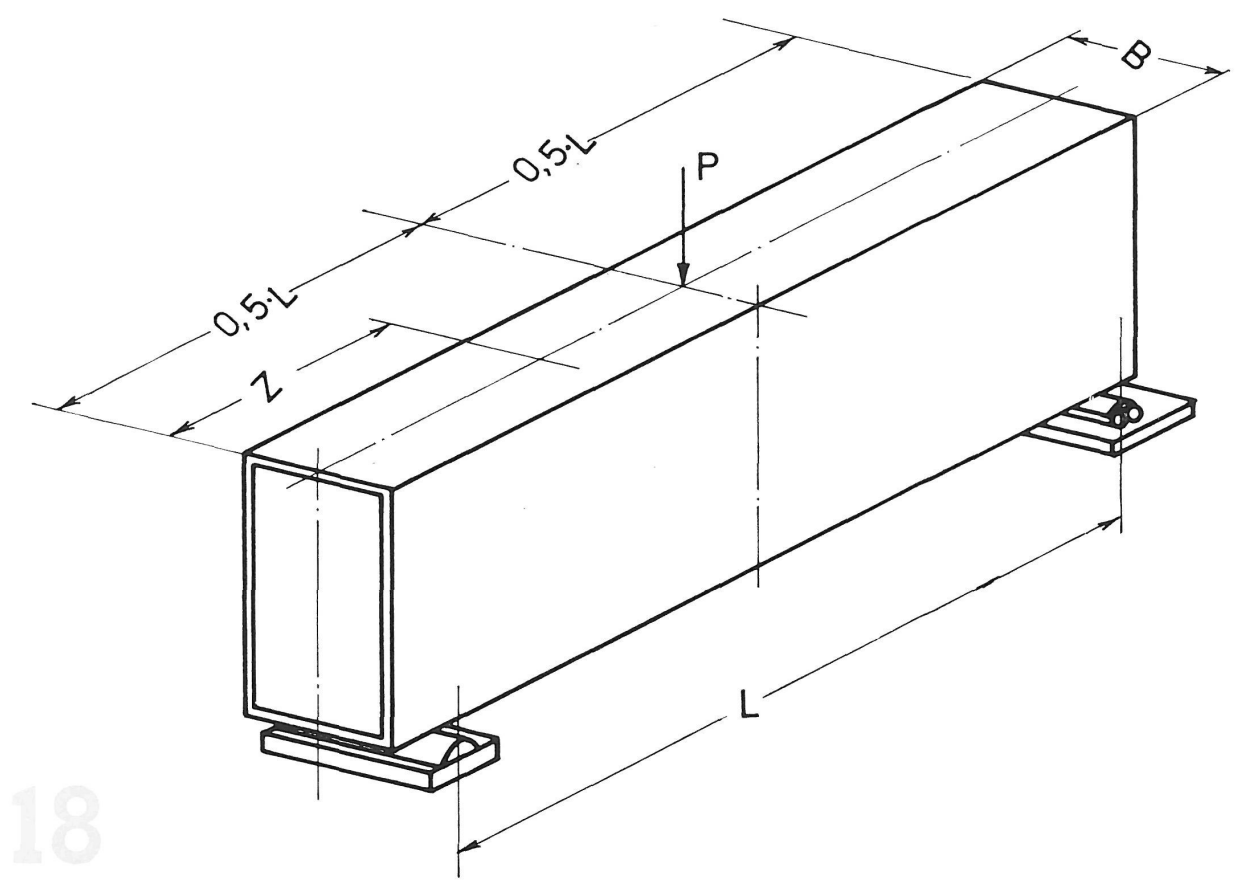

En la sección $z=0$ la tensión $\sigma=0$ y, por consiguiente, $D_{2}=0$.

En el centro del vano:

$$
\left(\frac{d \sigma}{d z}\right)_{z=0,5 \cdot L}=\gamma \cdot\left(\frac{d M(z)}{d z}\right)_{z=0,5 \cdot L}
$$

y

$$
u \cdot D_{1} \cdot \operatorname{ch} u(0,5 \cdot L)+0,5 \cdot P \cdot \frac{\eta}{u^{2}}=\gamma \cdot(0,5 \cdot P) ;
$$

de donde:

$$
D_{1}=\frac{P}{2 \cdot u \cdot \operatorname{ch} u(0,5 \cdot L)} \cdot\left(\gamma-\frac{\eta}{u^{2}}\right) .
$$

Como resultado obtenemos, para las tensiones normales en la platabanda, a la distancia $z$ del apoyo:

$$
\sigma=0,5 \cdot P \cdot\left[\left(\gamma-\frac{\eta}{u^{2}}\right) \cdot \frac{\operatorname{sh} u z}{u \cdot \operatorname{ch} u(0,5 \cdot L)}+\frac{\eta}{u^{2}} \cdot z \mid,\right.
$$

o en otra notación:

$$
\sigma=\frac{P}{2 \cdot B \cdot H \cdot t}\left[1+0,875 \cdot \frac{B \cdot \operatorname{sh} u z}{z \cdot \operatorname{ch} u(0,5 \cdot L)}\right]
$$


Recordando la simplificación de partida, que las tensiones normales son absorbidas exclusivamente por las platabandas, llegamos a la siguiente expresión para las tensiones normales originadas por la flexión libre:

$$
\sigma_{\text {fl. li. }}=\frac{M(z)}{J_{x}} \cdot y=\frac{P \cdot z}{2 \cdot B \cdot H \cdot t}
$$

y, en consecuencia, podemos escribir la ecuación [26] como sigue:

$$
\sigma=\sigma_{f l l l i}(1+K)
$$

en la que

$$
K=0,875 \cdot \frac{B \cdot \operatorname{sh} u z}{z \cdot \operatorname{ch} u(0,5 \cdot L)}=0,875 \cdot \frac{B \cdot \operatorname{sh} \frac{4 \cdot z}{B}}{z \cdot \operatorname{ch} \frac{2 \cdot L}{B}}
$$

es un coeficiente que caracteriza el grado de sobresolicitación de la platabanda en su unión al alma, punto en el cual alcanzan su valor máximo las tensiones secundarias.

En la viga simple de un solo vano, como era de esperar, el coeficiente $K$ alcanza su valor máximo en la sección del centro del vano, donde:

$$
K_{\max }=1,75 \cdot \frac{B \cdot \operatorname{sh} u(0,5 \cdot L)}{L \cdot \operatorname{ch} u(0,5 \cdot L)}=1,75 \cdot \frac{B}{L} \cdot \frac{\operatorname{sh} \frac{2 \cdot L}{B}}{\operatorname{ch} \frac{2 \cdot L}{B}}
$$

Recordando que, con valores elevados del argumento $X$, las funciones hiperbólicas

$$
\operatorname{sh} x \approx \operatorname{ch} x \approx 0,5 \cdot e^{x},
$$

podemos escribir que:

$$
K_{\max }=1,75 \cdot \frac{B}{L} \%
$$

El valor de las tensiones normales, en el punto de intersección de la platabanda con un alma, se obtiene introduciendo $\bar{\omega}=1$ en la expresión [23]:

$$
\sigma_{1}=\frac{M(z)}{J_{x}} \cdot \frac{H}{2}+\varphi(z)=\frac{M(z)}{J_{x}} \cdot \frac{H}{2} \cdot(1+K)
$$

siendo, para este punto:

$$
\varphi(z)=\frac{M(z)}{J_{x}} \cdot \frac{H}{2} \cdot K
$$

Para obtener la expresión general de la tensión normal en cualquiera de las fibras de la sección transversal de la platabanda debemos introducir en la [23] la última expresión de $p(z)$ :

$$
\sigma=\frac{M(z)}{J_{x}} \cdot \frac{H}{2} \cdot(1+K \cdot \bar{\omega})
$$

pero debemos calcular el valor de $K$ por la [27], y el del corrimiento $\bar{\omega}$ por la [24]. 
La expresión [27] permite hacernos una idea sobre las dimensiones de la extensión de la zona a lo largo del eje $Z$, en la cual se inducen tensiones secundarias importantes de coacción. En el gráfico de la figura 19, que tomamos de la referencia ${ }^{7}$, presentamos algunos valores de $K$ en función de $0,5 \cdot L / B$. Vemos que, cuanto mayor sea el ancho relativo de la viga en cajón, tanto mayor será la longitud $(0,5 \cdot L-z)$ de la zona sobre la cual se extiende la influencia de las tensiones secundarias de coacción. Esta zona se extiende, a cada lado de la sección del centro del vano, sobre una longitud aproximadamente igual al ancho $B$ de la platabanda. Aún cuando en la realidad en las vigas en cajón el número de diafragmas es discreto, las expresiones teóricas presentadas se pueden aplicar en la práctica, puesto que han sido avaladas por los resultados de la investigación experimental.

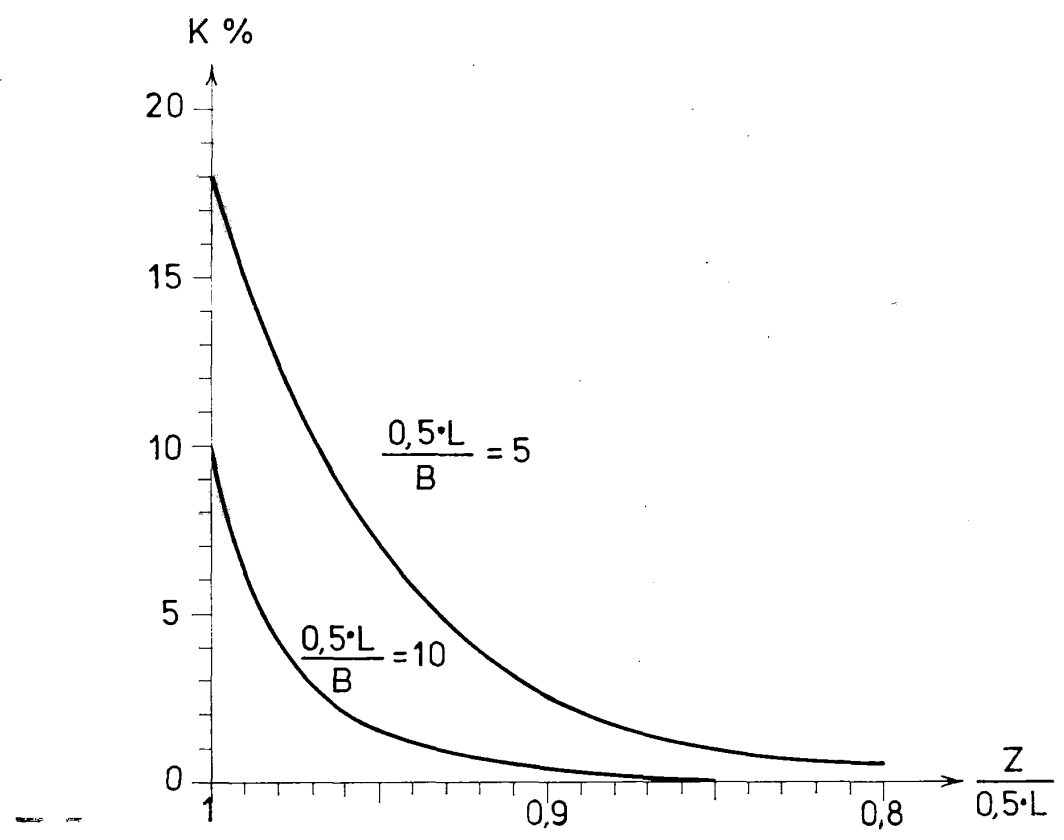

En la figura 20, que hemos extraído de la referencia ${ }^{6}$, presentamos el diagrama espacial de las tensiones normales, medidas en las secciones I a V de la platabanda superior de una viga, de sección en cajón ancho, que salva un vano de 4,0 $\mathrm{m}$ de luz entre apoyos y soporta una carga concentrada en el centro del mismo.

El valor calculado del coeficiente de sobresolicitación $K$ es:

$$
K=1,75 \cdot \frac{B}{L} \%=1,75 \cdot \frac{120}{400} \%=52,5 \%
$$

que coincide prácticamente con el valor $55 \%$, obtenido a partir de los siguientes resultados de los ensayos:

$$
\frac{\sigma_{\max }}{\sigma_{\min }}=2,14 ; \frac{\sigma_{\max }}{\sigma_{\operatorname{med}}}=1,33 ; \frac{\sigma_{\min }}{\sigma_{\operatorname{med}}}=0,2
$$

La figura 21, tomada de la misma referencia ${ }^{6}$, muestra la distribución de tensiones normales en la sección de la platabanda de una viga-carril de un solo vano, de $20,0 \mathrm{~m}$ de luz entre apoyos, que soporta dos cargas concentradas de $27.000 \mathrm{kgf}$ cada una, separadas $3,2 \mathrm{~m}$ una de otra. 


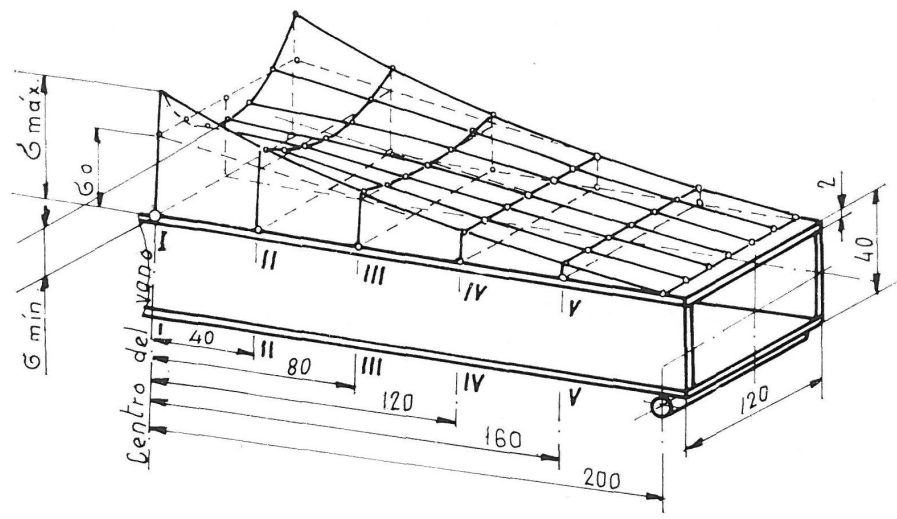

(2) (1)

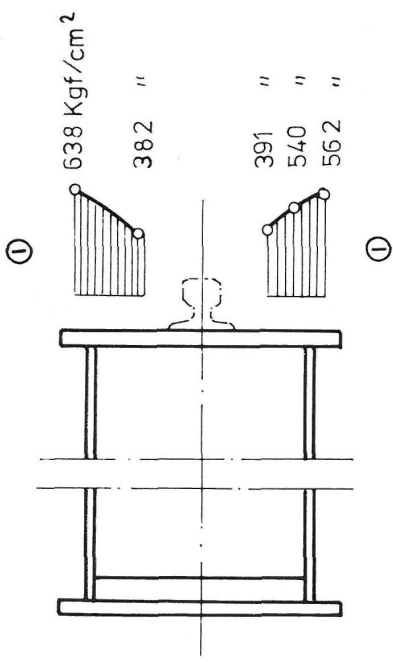

\section{Tensiones normales secundarias de la torsión coartada}

No quisiéramos terminar este artículo sin completarlo con la recopilación de las expresiones finales de la teoría de Vlasov de la flexión coartada. Puesto que, aun cuando el tema es bien conocido por el Ingeniero de Caminos, la información que presentamos a continuación podrá, sin embargo, ser de utilidad práctica para el Técnico que se inicia en el proyecto de estructuras metálicas especiales.

Al determinar el estado de tensiones en el caso de la torsión coartada, debemos superponer sobre las tensiones de la flexión y torsión simples, calculadas a partir de la hipótesis de Bernouilli, los valores de las tensiones normales secundarias inducidas por la coacción de la torsión. Tensiones secundarias que son importantes en el caso de los perfiles de sección abierta.

Las tensiones tangenciales secundarias, $\tau_{\omega}$, inducidas por la coacción de la torsión alcanzan, en general, valores muy inferiores a los de la torsión pura, por lo cual se pueden despreciar en los cálculos prácticos.

Distinguiremos dos casos bien diferenciados, como son: la viga de sección abierta y la viga de sección cerrada.

En el caso concreto de la viga de sección abierta el valor de la tensión normal secundaria, debida a la coacción de la torsión, según Vlasov $^{8}$, se calcula, para un punto cualquiera de la sección, por la expresión:

$$
\sigma_{\omega}=\frac{B_{\omega} \cdot \omega}{J_{\omega}}
$$

donde $B_{\omega}=E \cdot J_{\omega} \cdot \varphi^{\prime \prime}$ es el bimomento interno de flexión con torsión, que es función, como vemos, del ángulo de torsión $\varphi$, el cual depende, a su vez, de la coordenada $z$ de la sección. El valor de $B_{\omega}$ se puede determinar también, a partir de la ecuación diferencial siguiente:

en la que:

$$
B^{\prime \prime}{ }_{\omega}-K^{2} \cdot B_{\omega}=-m
$$

$m=m(z)=$ Intensidad de los momentos torsores exteriores.

$K=\sqrt{\frac{G \cdot J_{t}}{E \cdot J_{\omega}}}=$ Característica de flexión con torsión de la viga.

$\omega=$ Area sectorial principal (o coordenada sectorial) del punto, calculada con el polo de referencia situado en el centro de torsión de la sección.

$J_{\omega}=\int_{\dot{A}} \omega^{2} \cdot d A=$ Momento sectorial de inercia de la sección. 
Al determinar las dos constantes de integración de la solución general de la ecuación [31], debemos recordar, con respecto al alabeo, que las condiciones en cada uno de los dos extremos pueden ser:

$$
\begin{aligned}
B_{\omega} & =0, \text { cuando no existe coacción alguna al alabeo; } \\
B^{\prime}{ }_{\omega} & =M, \text { cuando el alabeo es imposible. }
\end{aligned}
$$

Para el mismo punto de la sección, la tensión tangencial de coacción de la torsión se calcula por la expresión siguiente:

$$
\tau_{\omega}=\frac{M_{\omega} \cdot S_{\omega}}{J_{\omega} \cdot t}
$$

siendo:

$$
\begin{aligned}
& M_{\omega}=B_{\omega}^{B^{\prime}} \quad=\text { Momento torsor de la coacción. } \\
& S_{\omega}=\int_{0}^{S} \omega \cdot d A=\text { Momento sectorial estático. }
\end{aligned}
$$

La tensión tangencial de torsión libre es, como siempre:

$$
\tau_{*}=\frac{M_{*} \cdot t}{2 \cdot J_{t}}
$$

donde $J_{t}$ es el módulo de torsión de la sección.

Entre el momento torsor exterior $M$, el interior de torsión libre $M_{*}$ y el mismo de la coacción existe la siguiente relación:

$$
M=M_{*}+M_{\omega}
$$

En la tabla 5 hemos recopilado los diferentes tipos de sustentación y las correspondientes condiciones en el extremo, mientras que en la tabla 6 recogemos las expresiones finales de los bimomentos internos, en función del esquema de carga y del tipo de viga, siendo ésta siempre de perfil abierto. La tabla 7 reúne las expresiones de los coeficientes que aparecen en la 6, así como sus valores numéricos. En el cálculo de la viga continua, para resolver la ecuación de los tres bimomentos, también es interesante conocer los valores de los coeficientes $r$ y $s$.

Los tres últimos casos de carga corresponden a la acción de cargas en forma de bimomentos concentrados.

En consecuencia, el valor máximo de la tensión normal secundaria en el punto más solicitado de la sección de la viga se calcula como sigue:

$$
\sigma_{\omega \max }=\frac{B_{\omega} \cdot \omega_{\max }}{\mathbf{J}_{\omega}}
$$

En el caso de la viga de sección en cajón (o perfil cerrado) las tensiones normales inducidas por la torsión coartada se determinan haciendo uso de una expresión análoga a la [30], cual es:

$$
\sigma_{\omega}=\frac{B_{\omega}}{J_{\hat{\omega}}} \cdot \hat{\omega}
$$


TABLA 5

\begin{tabular}{|c|c|c|}
\hline Tipo de sustentación & Esquema & Condiciones en el extremo \\
\hline $\begin{array}{l}\text { Empotramiento completo. } \\
\text { El giro, alrededor del eje } \\
\text { z, y el alabeo de la sec- } \\
\text { ción están impedidos. }\end{array}$ & & $\begin{array}{l}\varphi=0 ; \\
\varphi^{\prime}=0\end{array}$ \\
\hline $\begin{array}{l}\text { El giro está impedido; } \\
\text { el alabeo es libre }\end{array}$ & 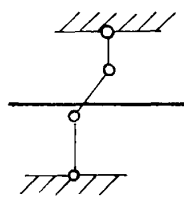 & $\begin{array}{l}\varphi=0 ; \\
\varphi^{\prime \prime}=0\end{array}$ \\
\hline $\begin{array}{l}\text { El giro es libre; } \\
\text { el alabeo está impedido }\end{array}$ & & $\begin{array}{l}\varphi^{\prime}=0 ; \\
\varphi^{\prime \prime \prime}=0\end{array}$ \\
\hline $\begin{array}{l}\text { El giro es libre; } \\
\text { el alabeo está impedido. } \\
\text { En el extremo actúa un mo- } \\
\text { mento torsor concentrado }\end{array}$ & M & $\begin{aligned} \varphi^{\prime} & =0 ; \\
\varphi^{\prime \prime \prime} & =-\frac{M}{E \cdot J_{\omega}}\end{aligned}$ \\
\hline Extremo libre & & $\begin{array}{l}\varphi^{\prime \prime}=0 ; \\
\varphi^{\prime \prime \prime}=k^{2} \cdot \varphi^{\prime}\end{array}$ \\
\hline $\begin{array}{l}\text { En el extremo libre actúa } \\
\text { un momento torsor concen- } \\
\text { trado }\end{array}$ & $M$ & $\begin{array}{c}\varphi^{\prime \prime}=0 ; \\
\varphi^{\prime \prime \prime}-k^{2} \cdot \varphi^{\prime}=-\frac{M}{E \cdot J_{\omega}}\end{array}$ \\
\hline
\end{tabular}


TABLA 6

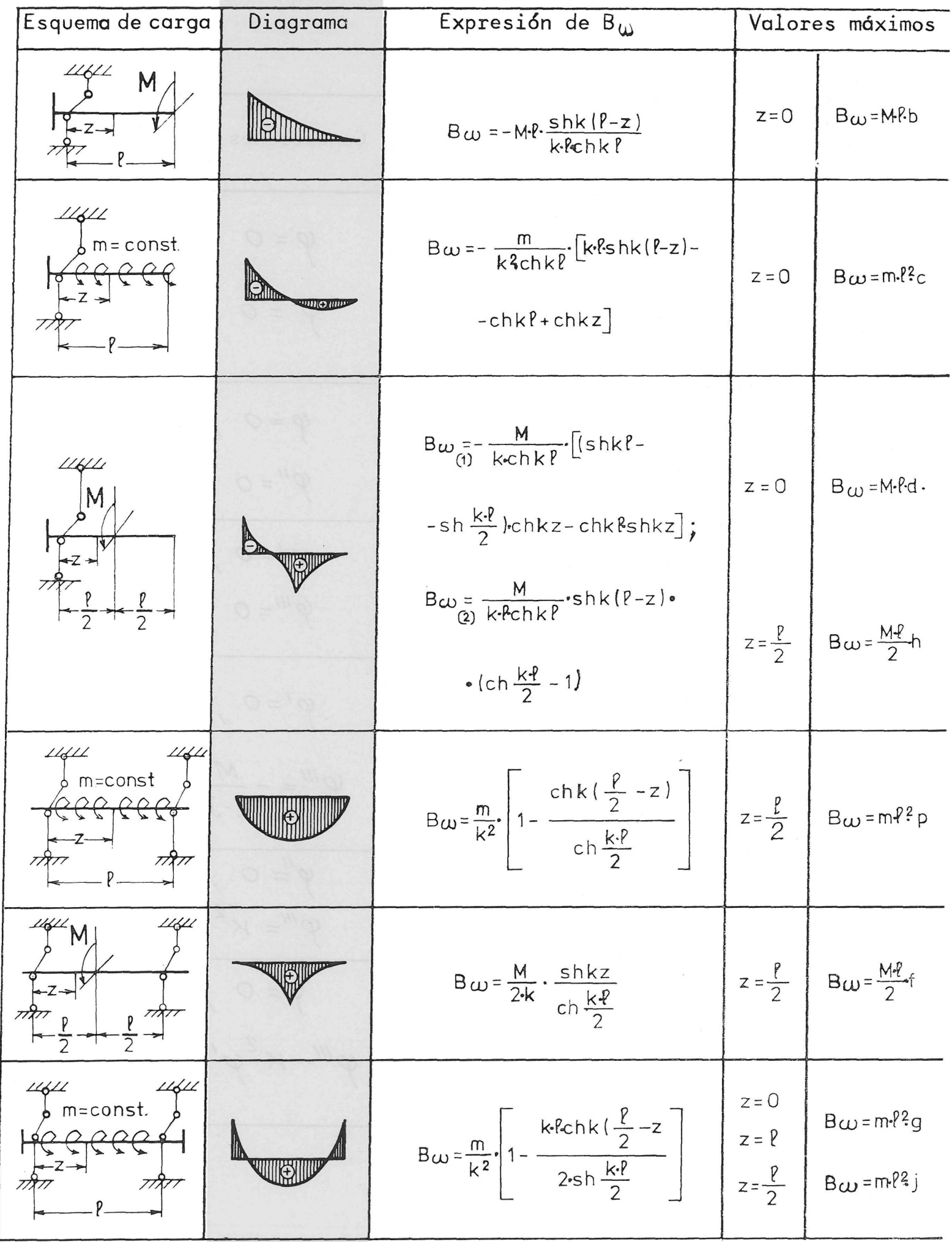


TABLA 6 (Cont.)

\begin{tabular}{|c|c|c|c|c|}
\hline Esquema de carga & Diagrama & Expresión de $B_{\omega}$ & Valor & s máximos \\
\hline 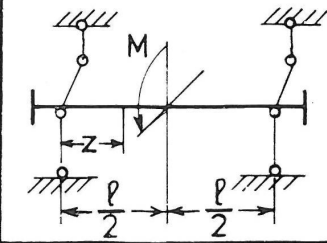 & & $B_{\omega}=\frac{M}{(1)} \frac{\operatorname{chk} z-\operatorname{chk}\left(\frac{l}{2}-z\right)}{\operatorname{sh} \frac{k \cdot l}{2}}$ & $\begin{array}{l}z=0 \\
z=\frac{l}{2} \\
z=P\end{array}$ & $B_{\omega}=\frac{M \cdot P}{2} \cdot n$ \\
\hline 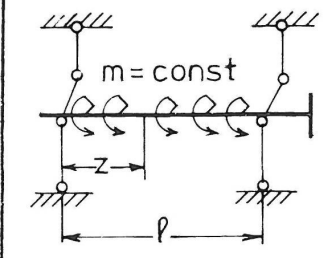 & & $\begin{array}{l}B_{\omega}=\frac{m}{k^{2}} \cdot(1-\operatorname{chk} z+ \\
\left.+\frac{1+k \cdot P \cdot \operatorname{sh} k P-\operatorname{chk} P-\frac{k^{2} \cdot P^{2}}{2}}{k \cdot \operatorname{ch} k P-\operatorname{sh} k l} \operatorname{shk} z\right)\end{array}$ & $z=\ell$ & $B_{\omega}=\frac{m \cdot P^{2}}{2} w$ \\
\hline 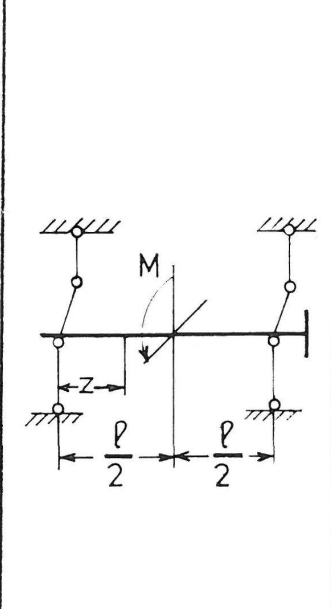 & & $\begin{array}{l}B_{\omega}=\frac{M}{(1) k} \cdot \frac{1}{k \cdot P \cdot c h k l-s h k P} \cdot \\
\cdot\left(k P c h \frac{k P}{2}-\operatorname{sh} \frac{k \cdot P}{2}-\frac{k \cdot P}{2}\right) \cdot s h k z ; \\
B_{\omega}=\frac{M}{(2)} \cdot\left[\frac{\operatorname{sh} k z}{k \cdot P \operatorname{chk} P-\operatorname{shk} P} \cdot\right. \\
\cdot\left(k \cdot P \cdot \operatorname{ch} \frac{k \cdot P}{2}-\operatorname{sh} \frac{k \cdot P}{2}-\frac{k \cdot P}{2}\right)- \\
\left.-\operatorname{shk}\left(z-\frac{l}{2}\right)\right]\end{array}$ & $z=\frac{p}{2}$ & $\begin{array}{l}B_{\omega}=\frac{M \cdot \ell}{2} \cdot v \\
B_{\omega}=\frac{M \ell}{2} \cdot u\end{array}$ \\
\hline 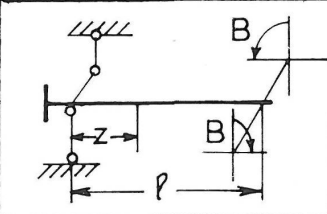 & 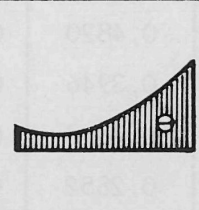 & $B_{\omega}=-B \cdot \frac{\operatorname{chk} z}{\operatorname{chk} P}$ & $\begin{array}{l}z=0 \\
z=l\end{array}$ & $\begin{array}{l}\mathrm{B}_{\omega}=\mathrm{Ba} \\
\mathrm{B}_{\omega}=\mathrm{B}\end{array}$ \\
\hline 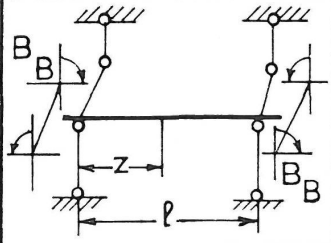 & indm & $\begin{aligned} B_{\omega}= & -B_{A} \cdot \frac{\operatorname{shk}(l-z)}{\operatorname{shkl}}- \\
& -B_{B} \cdot \frac{\operatorname{sh} k z}{\operatorname{shk} l}\end{aligned}$ & $\begin{array}{l}z=0 \\
z=l\end{array}$ & $\begin{array}{l}\mathrm{B}_{\omega}=\mathrm{B}_{\mathrm{A}} \\
\mathrm{B}_{\omega}=\mathrm{B}_{\mathrm{B}}\end{array}$ \\
\hline 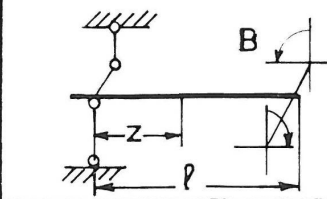 & & $B_{\omega}=-B \cdot \frac{\operatorname{shkz}}{\operatorname{shkl}}$ & $z=l$ & $\mathrm{~B}_{\omega}=\mathrm{B}$ \\
\hline
\end{tabular}


TABLA 7. Expresiones de los coeficientes

$$
\begin{array}{ll}
r=\frac{k \cdot l \cdot \operatorname{ch} k l-\operatorname{sh} k l}{k^{2} \cdot l^{2} \cdot \operatorname{sh} k l} \quad ; \quad t=\frac{k \cdot l \cdot \operatorname{sh} k l-2 \cdot \operatorname{ch} k l+2}{K^{3} \cdot l^{3} \cdot \operatorname{sh} k l} ; b=\frac{\text { th } k l}{k \cdot l} ; \\
c=\frac{K \cdot l \cdot \operatorname{sh} k l-\operatorname{ch} k l+1}{k^{2} \cdot l^{2} \cdot \operatorname{ch} k l} ; s=\frac{\operatorname{sh} k l-k \cdot l}{k^{2} \cdot l^{2} \cdot \operatorname{sh} k l} & ; p=\frac{\operatorname{ch} \frac{k \cdot l}{2}-1}{k^{2} \cdot l \cdot \operatorname{ch} \frac{k \cdot l}{2}} ; \\
a=\frac{1}{\operatorname{ch} k l} & ; f=\frac{\operatorname{ch} k l-1}{k \cdot l \cdot \operatorname{sh} k l} .
\end{array}
$$

\begin{tabular}{|c|c|c|c|c|c|c|c|c|}
\hline$k \cdot l$ & $r$ & $s$ & $t$ & $p$ & $a$ & $b$ & $c$ & $f$ \\
\hline 0,0 & 0,3333 & 0,1667 & 0,08333 & 0,125 & 1,0000 & 1,0000 & 0,5000 & 0,5000 \\
\hline 0,1 & 0,3331 & 0,1665 & 0,08325 & 0,1248 & 0,9960 & 0,9972 & 0,4988 & 0,4996 \\
\hline 0,2 & 0,3324 & 0,1659 & 0,08294 & 0,1244 & 0,9803 & 0,9869 & 0,4951 & 0,4983 \\
\hline 0,3 & 0,3314 & 0,1649 & 0,08258 & 0,1239 & 0,9566 & 0,9710 & 0,4891 & 0,4963 \\
\hline 0,4 & 0,3298 & 0,1636 & 0,08202 & 0,1229 & 0,9250 & 0,9499 & 0,4812 & 0,4934 \\
\hline 0,5 & 0,3279 & 0,1620 & 0,08129 & 0,1218 & 0,8868 & 0,9242 & 0,4715 & 0,4898 \\
\hline 0,6 & 0,3256 & 0,1599 & 0,08044 & 0,1205 & 0,8435 & 0,8951 & 0,4605 & 0,4855 \\
\hline 0,7 & 0,3229 & 0,1576 & 0,07943 & 0,1189 & 0,7967 & 0,8634 & 0,4485 & 0,4805 \\
\hline 0,8 & 0,3199 & 0,1550 & 0,07825 & 0,1172 & 0,7477 & 0,8301 & 0,4358 & 0,4749 \\
\hline 0,9 & 0,3166 & 0,1522 & 0,07709 & 0,1153 & 0,6977 & 0,7959 & 0,4228 & 0,4688 \\
\hline 1,0 & 0,3130 & 0,1491 & 0,07577 & 0,1132 & 0,6480 & 0,7616 & 0,4097 & 0,4621 \\
\hline 1,2 & 0,3052 & 0,1424 & 0,07285 & 0,1087 & 0,5522 & 0,6947 & 0,3838 & 0,4475 \\
\hline $1 ; 4$ & 0,2966 & 0,1351 & 0,06970 & 0,1037 & 0,4649 & 0,6324 & 0,3594 & 0,4317 \\
\hline 1,6 & 0,2875 & 0,1275 & 0,06639 & 0,0985 & 0,3880 & 0,5760 & 0,3370 & 0,4150 \\
\hline 1,8 & 0,2781 & 0,1198 & 0,06299 & 0,0933 & 0,3218 & 0,5260 & 0,3167 & 0,3979 \\
\hline 2,0 & 0,2687 & 0,1121 & 0,05960 & 0,0880 & 0,2658 & 0,4820 & 0,2985 & 0,3808 \\
\hline 2,5 & 0,2452 & 0,0939 & 0,05142 & 0,0753 & 0,1631 & 0,3946 & 0,2607 & 0,3393 \\
\hline 3,0 & 0,2239 & 0,0778 & 0,04406 & 0,0639 & 0,0993 & 0,3317 & 0,2316 & 0,3017 \\
\hline 3,5 & 0,2046 & 0,0644 & 0,03772 & 0,0541 & 0,0603 & 0,2852 & 0,2085 & 0,2690 \\
\hline 4,0 & 0,1877 & 0,0533 & 0,03237 & 0,0459 & 0,0366 & 0,2498 & 0,1896 & 0,2410 \\
\hline 5,0 & 0,1600 & 0,0373 & 0,02421 & 0,0335 & 0,0135 & 0,2000 & 0,1605 & 0,1973 \\
\hline 6,0 & 0,1389 & 0,0270 & 0,01856 & 0,0250 & $0,496 \cdot 10^{-2}$ & 0,1667 & 0,1390 & 0,1658 \\
\hline 7,0 & 0,1225 & 0,0201 & 0,01459 & 0,0192 & $0,182 \cdot 10^{-2}$ & 0,1429 & 0,1225 & 0,1426 \\
\hline 8,0 & 0,1094 & 0,0155 & 0,01172 & 0,0151 & $0,671 \cdot 10^{-3}$ & 0,1251 & 0,1094 & 0,1249 \\
\hline 9,0 & 0,0988 & 0,0123 & 0,00960 & 0,0121 & $0,247 \cdot 10^{-3}$ & 0,1111 & 0,0988 & 0,1111 \\
\hline 10,0 & 0,0900 & 0,0100 & 0,00800 & 0,00987 & $0,908 \cdot 10^{-4}$ & 0,1000 & 0,0900 & 0,1000 \\
\hline 11,0 & 0,0826 & 0,00826 & 0,00676 & 0,00820 & $0,334 \cdot 10^{-4}$ & 0,0909 & 0,0826 & 0,0909 \\
\hline 12,0 & 0,0764 & 0,00694 & 0,00579 & 0,00691 & $0,123 \cdot 10^{-4}$ & 0,0833 & 0,0764 & 0,0833 \\
\hline 13,0 & 0,0710 & 0,00592 & 0,00501 & 0,00590 & $0,452 \cdot 10^{-5}$ & 0,0769 & 0,0710 & 0,0769 \\
\hline 14,0 & 0,0663 & 0,0051 & 0,00437 & 0,00509 & $0,166 \cdot 10^{-5}$ & 0,0714 & 0,0663 & 0,0714 \\
\hline 15,0 & 0,0622 & 0,00445 & 0,00385 & 0,00441 & $0,612 \cdot 10^{-6}$ & 0,0667 & 0,0622 & 0,0667 \\
\hline
\end{tabular}

VALORES NUMERICOS DE LOS COEFICIENTES 
TABLA 7a. Expresiones de los coeficientes

$d=\frac{\operatorname{sh} k l-\operatorname{sh} \frac{k \cdot l}{2}}{k \cdot l \cdot \operatorname{ch} k l} \quad ; v=\frac{k \cdot l \cdot \operatorname{sh} k l-\operatorname{ch} k l+1-k \cdot l \cdot \operatorname{sh} \frac{k \cdot l}{2}}{k \cdot l \cdot(k \cdot l \cdot \operatorname{ch} k l-\operatorname{sh} k l)}$

$u=\frac{\operatorname{sh} k l-2 \cdot \operatorname{sh} \frac{k \cdot l}{2}}{k \cdot l \cdot \operatorname{ch} k l-\operatorname{sh} k l} \quad ; g=\frac{\frac{k \cdot l}{2} \cdot(\operatorname{ch} k l+1)-\operatorname{sh} k l}{k^{2} \cdot l^{2} \cdot \operatorname{sh} k l} \quad ; \quad h=\frac{\operatorname{sh} k l-2 \cdot \operatorname{sh} \frac{k \cdot l}{2}}{k \cdot l \cdot \operatorname{ch} k l} ;$

$j=\frac{\operatorname{sh} k l-k \cdot l \cdot \operatorname{ch} \frac{k \cdot l}{2}}{k^{2} \cdot l^{2} \cdot \operatorname{sh} k l} ; w=\frac{k \cdot l \cdot \operatorname{ch} k l-2 \cdot \operatorname{ch} k l+2}{k \cdot l \cdot(K \cdot l \cdot \operatorname{ch} k l-\operatorname{sh} k l)} ; n=\frac{\operatorname{sh} k l-2 \cdot \operatorname{sh} \frac{k \cdot l}{2}}{k \cdot l \cdot(\operatorname{sh} k l-1)} ;$

VALORES NUMERICOS DE LOS COEFICIENTES

\begin{tabular}{|c|c|c|c|c|c|c|c|c|}
\hline$k \cdot l$ & $d$ & $j$ & $v$ & $w$ & $t i$ & $h$ & g & $n$ \\
\hline 0,0 & 0,5000 & 0,04167 & 0,3125 & 0,2500 & 0,3750 & 0,0000 & 0,08333 & 0,2500 \\
\hline 0,1 & 0,4990 & 0,04165 & 0,0124 & 0,2499 & 0,3748 & 0,00124 & 0,08332 & 0,2499 \\
\hline 0,2 & 0,4959 & 0,04162 & 0,3123 & 0,2496 & 0,3744 & 0,00491 & 0,08328 & 0,2496 \\
\hline 0,3 & 0,4909 & 0,04156 & 0,3115 & 0,2492 & 0,3738 & 0,0108 & 0,08321 & 0,2495 \\
\hline 0,4 & 0,4843 & 0,04147 & 0,3107 & 0,2486 & 0,3728 & 0,0187 & 0,08312 & 0,2492 \\
\hline 0,5 & 0,4762 & 0,04136 & 0,3099 & 0,2479 & 0,3715 & 0,0281 & 0,08300 & 0,2487 \\
\hline 0,6 & 0,4670 & 0,04123 & 0,3085 & 0,2470 & 0,3700 & 0,0388 & 0,08284 & 0,2482 \\
\hline 0,7 & 0,4569 & 0,04108 & 0,3071 & 0,2460 & 0,3683 & 0,0503 & 0,08266 & 0,2475 \\
\hline 0,8 & 0,4461 & 0,04090 & 0,3056 & 0,2446 & 0,3663 & 0,0622 & 0,08246 & 0,2467 \\
\hline 0,9 & 0,4351 & 0,0407 & 0,3038 & 0,2435 & 0,3640 & 0,0743 & 0,08223 & 0,2459 \\
\hline 1,0 & 0,4239 & 0,0408 & 0,3018 & 0,2420 & 0,3616 & 0,0862 & 0,08198 & 0,2449 \\
\hline 1,2 & 0,4017 & 0,03998 & 0,2974 & 0,2387 & 0,3560 & 0,1087 & 0,08140 & 0,2428 \\
\hline 1,4 & 0,3805 & 0,03940 & 0,2924 & 0,2350 & 0,3497 & 0,1286 & 0,08073 & 0,2403 \\
\hline 1,6 & 0,3607 & 0,03875 & 0,2869 & 0,2309 & 0,3428 & 0,1453 & 0,07999 & 0,2375 \\
\hline 1,8 & 0,3425 & 0,03804 & 0,2809 & 0,2265 & 0,3354 & 0,1590 & 0,07915 & 0,2344 \\
\hline 2,0 & 0,3258 & 0,03727 & 0,2747 & 0,2218 & 0,3275 & 0,1696 & 0,07825 & 0,2311 \\
\hline 2,5 & 0,2902 & 0,03515 & 0,2581 & 0,2095 & 0,3067 & 0,1857 & 0,07577 & 0,2218 \\
\hline 3,0 & 0,2612 & 0,03284 & 0,2411 & 0,1968 & 0,2853 & 0,1907 & 0,07303 & 0,2117 \\
\hline 3,5 & 0,2371 & 0,03044 & 0,2244 & 0,1844 & 0,2644 & 0,1890 & 0,07012 & 0,2011 \\
\hline 4,0 & 0,2166 & 0,02803 & 0,2085 & 0,1725 & 0,2445 & 0,1834 & 0,06717 & 0,1904 \\
\hline 5,0 & 0,1837 & 0,02347 & 0,1803 & 0,1513 & 0,2092 & 0,1674 & 0,06136 & 0,1697 \\
\hline 6,0 & 0,1584 & 0,01946 & 0,1569 & 0,1337 & 0,1801 & 0,1501 & 0,05597 & 0,1509 \\
\hline 7,0 & 0,1385 & 0,01609 & 0,1379 & 0,1191 & 0,1566 & 0,1342 & 0,05115 & 0,1345 \\
\hline 8,0 & 0,1227 & 0,01333 & 0,1224 & 0,1071 & 0,1376 & 0,1204 & 0,04692 & 0,1205 \\
\hline 9,0 & 0,1099 & 0,01111 & 0,1097 & 0,0972 & 0,1222 & 0,1086 & 0,04322 & 0,1087 \\
\hline 10,0 & 0,0993 & 0,00933 & 0,0993 & 0,0889 & 0,1096 & 0,0987 & 0,04001 & 0,0987 \\
\hline 11,0 & 0,0905 & 0,00789 & 0,0905 & 0,0818 & 0,0992 & 0,0902 & 0,03719 & 0,0902 \\
\hline 12,0 & 0,0831 & 0,00674 & 0,0831 & 0,0758 & 0,0905 & 0,0829 & 0,03475 & 0,0829 \\
\hline 13,0 & 0,0768 & 0,00580 & 0,0768 & 0,0705 & 0,0831 & 0,0767 & 0,03254 & 0,0767 \\
\hline 14,0 & 0,0714 & 0,00504 & 0,0714 & 0,0659 & 0,0768 & 0,0713 & 0,03061 & 0,0713 \\
\hline 15,0 & 0,0666 & 0,00441 & 0,0666 & 0,0619 & 0,0713 & 0,0666 & 0,02889 & 0,0666 \\
\hline
\end{tabular}


siendo en este caso para los perfiles cerrados:

$$
\hat{\omega}=\omega-\frac{A_{c}}{s_{c}^{\prime}} \cdot s^{\prime}
$$

donde:

$$
\begin{aligned}
\omega & =\text { Coordenada sectorial del punto. } \\
A_{c}= & \text { Al doble del área comprendida dentro del contorno de la sección de } \\
& \text { la viga. } \\
s_{c}^{\prime}=\sum \frac{s}{t}= & \begin{array}{l}
\text { Perímetro relativo del perfil, que comprende todo el desarrollo del con- } \\
\text { torno cerrado. }
\end{array} \\
s^{\prime}= & \text { Longitud relativa de cada uno de los lados del perímetro. } \\
s= & \text { Longitud del lado. }
\end{aligned}
$$

En el caso concreto de la viga en cajón (fig. 22), la expresión [38] toma la siguiente forma:

$$
\hat{\omega}=\omega-\frac{2 \cdot B \cdot H}{\sum \frac{s}{t}} \cdot \frac{s}{t}
$$

El valor del bimomento $B_{\omega}$ de flexión con torsión se calcula para cada sección de la viga de perfil cerrado a partir de la expresión siguiente:

$$
B^{\prime \prime}{ }_{\omega}-\mu \cdot K^{2} \cdot B_{\omega}=\mu \cdot m
$$

en la cual:

$$
\begin{aligned}
\mu=1-\frac{J_{t}}{J_{p}} & =\text { Coeficiente de alabeo de la sección, siendo: } \\
J_{t}=\frac{4 \cdot A^{2}}{\sum \frac{s}{t}} & =\text { Módulo de torsión de la sección. } \\
J_{p} & =\text { Su momento de inercia polar. }
\end{aligned}
$$

Vemos que la ecuación [40] en su composición es análoga a la [31] del perfil abierto; pero en este caso la característica de rigidez frente a la flexión con torsión es:

$$
K=\sqrt{\frac{G \cdot J_{t}}{E \cdot J_{\hat{\omega}}}}
$$
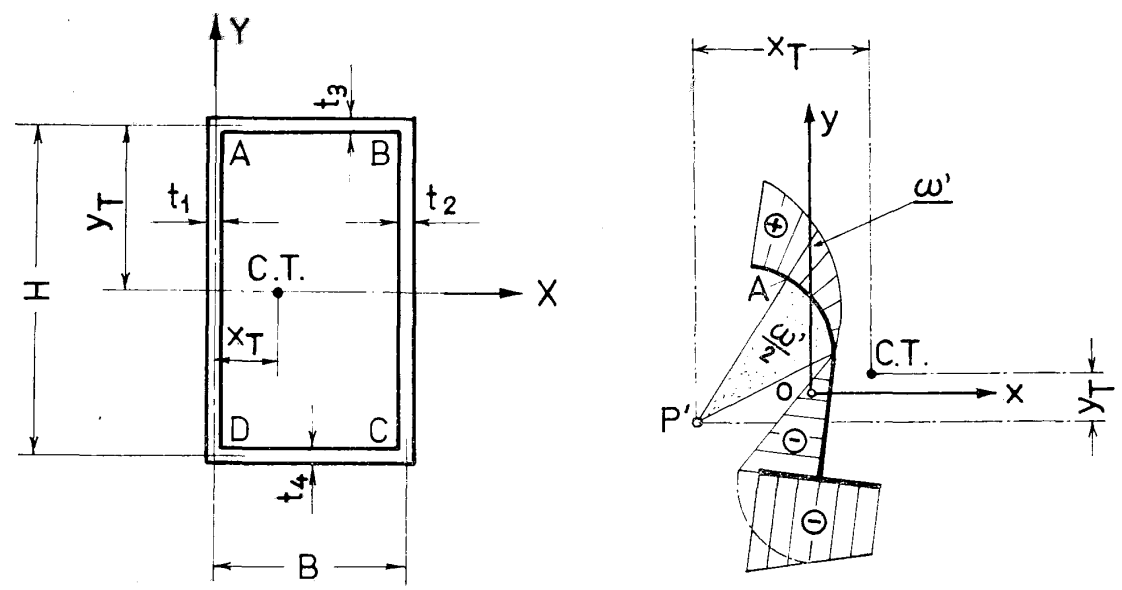
y el momento sectorial de inercia de la sección:

$$
J_{\hat{\omega}}=\int_{S} \hat{\omega}^{2} \cdot t \cdot d s,
$$

con su polo de referencia situado en el centro de torsión.

Para una sección cualquiera las tensiones normales, según la [36], son proporcionales a las ordenadas del diagrama de las $\hat{\omega}$, mientras que las $\sigma_{\omega}$, a lo largo de la viga, son función del diagrama del bimomento $B_{\omega}$, el cual depende de la ley de variación de la intensidad de los momentos torsores exteriores $m$ por unidad de longitud.

En el caso de los perfiles cerrados, para calcular los valores del bimomento $B_{\omega}$ de flexión con torsión se puede hacer uso de las expresiones de la tabla 6, previa realización de las siguientes transformaciones:

Primero: Multiplicar por $\mu$ los valores de los momentos torsores exteriores $m$.

Segundo: Introducir en las expresiones, en lugar del coeficiente $K$, su equivalente:

$$
K^{\prime}=\sqrt{\mu \cdot \frac{G \cdot J_{t}}{E \cdot J_{\hat{\omega}}}} .
$$

\section{Determinación práctica del centro de torsión en vigas-carriles}

Según se desprende de la información recopilada en el apartado anterior, nos es imprescindible, a la hora de proyectar el tipo de estructuras que indica el encabezamiento de este artículo, saber determinar con precisión la situación del centro de torsión de la sección de la viga.

Sin embargo, en la vida real no son muy raros los casos, en los que el proyectista no tiene una idea clara sobre este punto. Es típica la consideración de que, tanto en la viga-carril de sección doble te en alma llena, con viga horizontal de frenada también de alma llena, como en viga-carril de alma llena, pero con viga de frenado en celosía, el centro de torsión se localiza en el punto de intersección de las dos almas, cuales son la vertical y la horizontal.

Esta simplificación es prácticamente correcta en el primer caso, y, por consiguiente, válido su uso; pero es incorrecta en el segundo, con el agravante de permanecer del lado de la inseguridad.

Las coordenadas del centro de torsión de un perfil abierto se determinan, como es sabido, por las expresiones siguientes:

$$
X_{t}=\frac{\int_{s} \omega^{\prime} \cdot y \cdot t \cdot d s}{J_{x}} \quad ; \quad Y_{t}=\frac{\int_{S} \omega^{\prime} \cdot x \cdot t \cdot d s}{J_{y}}
$$

en las que:

$\omega^{\prime}=$ Area sectorial de la sección respecto a un polo arbitrario $P$.

$t=$ Espesor del alma.

$x$ e $y=$ Coordenadas de los puntos de la línea media del contorno del perfil respecto a los ejes principales de inercia.

$J_{x}, J_{y}$ son los momentos de inercia respecto a los mismos ejes principales. 
Las coordenadas $X_{t}$ e $Y_{t}$ se calculan a partir de un sistema cualquiera de ejes paralelos a los principales de inercia, con el origen arbitrariamente elegido, sirviendo éste de polo en la determinación de $\omega^{\prime}$ (fig. 23).

En la ${ }^{9}$ se trata el tema con detalle.

En el caso del perfil cerrado, como lo es toda viga-carril que salve luces superiores a los $15 \mathrm{~m}$ y que forme parte del camino de puentes-grúa de mediana fuerza y, sobre todo, de gran fuerza de elevación, las coordenadas del centro de torsión se pueden determinar partiendo de las expresiones deducidas para la viga de sección en cajón rectangular, con las cuatro caras de alma llena (fig. 22).

La solución exacta en el último caso es ${ }^{7}$ :

$X_{T}=B \cdot \frac{\left(\alpha_{1}+\alpha_{2}\right) \cdot\left\{\beta \cdot\left[\left(2 \cdot \eta \cdot \mu_{1}-3 \cdot \mu_{2}\right) \alpha_{2}-\eta \cdot \mu_{1} \cdot \alpha_{1}\right]+6 \cdot \eta \cdot \mu_{2} \cdot \mu_{3} \cdot\left[\alpha_{1}+\left(1+2 \cdot \mu_{1} \cdot \mu_{2}\right) \cdot \alpha_{2}\right]\right\}}{2 \cdot \beta \cdot\left[\mu_{1} \cdot(\eta+1) \cdot\left(\alpha_{1}^{2}-\alpha_{1} \cdot \alpha_{2}+\alpha_{2}^{2}\right)+3 \cdot\left(\alpha_{1}^{2} \cdot \mu_{3}+\alpha_{2}^{2} \cdot \mu_{2}\right)\right]}$

en la que:

$$
\left.\begin{array}{c}
\eta=\frac{t_{2}}{t_{1}} ; \quad \mu_{1}=\frac{H}{B} \quad ; \quad \mu_{2}=\frac{t_{3}}{t_{1}} ; \quad \mu_{3}=\frac{t_{4}}{t_{1}} \\
\alpha_{1}=\mu_{2}+\frac{\mu_{1}}{2} \cdot(\eta+1) \quad ; \quad \alpha_{2}=\mu_{3}+\frac{\mu_{1}}{2} \cdot(\eta+1) ; \\
\beta=\eta \cdot\left(\mu_{2}+\mu_{3}\right)+\mu_{1} \cdot \mu_{2} \cdot \mu_{3} \cdot(\eta+1) .
\end{array}\right]
$$

La coordenada vertical $Y_{t}$ del centro de torsión la determinamos empleando la [42], tras sustituir en ésta $H$ por $B$, y adoptar, en lugar de las [43], la siguiente notación:

$$
\eta=\frac{t_{4}}{t_{3}} \quad ; \quad \mu_{1}=\frac{B}{H} \quad ; \quad \mu_{2}=\frac{t_{2}}{t_{3}} \quad ; \quad \mu_{3}=\frac{t_{1}}{t_{3}} .
$$

También podemos emplear la expresión [42] en el caso de la viga en cajón con tres caras de alma llena y la cuarta en celosía o en viga Vierendeel. Para lo cual debemos sustituir el alma de celosía, o de montantes, por su equivalente energética en alma llena. Denominaremos alma llena equivalente al alma llena que, bajo la acción de una determinada carga, almacene una cantidad de energía potencial de cortadura igual a la energía de tracción (o compresión) que almacenan los elementos de la triangulación, bajo la misma carga, en el alma de celosía, o a la energía de flexión del pórtico múltiple bajo igual carga, en el caso de la viga Vierendeel.

A continuación determinamos el espesor del alma equivalente que sustituye a la de celosía o pórtico múltiple, en los ejemplos de las estructuras más simples, como son los que presentamos en la figura 25.

Partimos de un alma (una placa rectangular) sobre el contorno de la cual se distribuyen los esfuerzos tangenciales (fig. 24). De las condiciones de equilibrio se desprende que $q_{1}=q_{2}=$ $=q_{3}=q_{4}=q$, y que en cada lado del contorno los esfuerzos resultantes son $q \cdot a$ y $q \cdot b$.
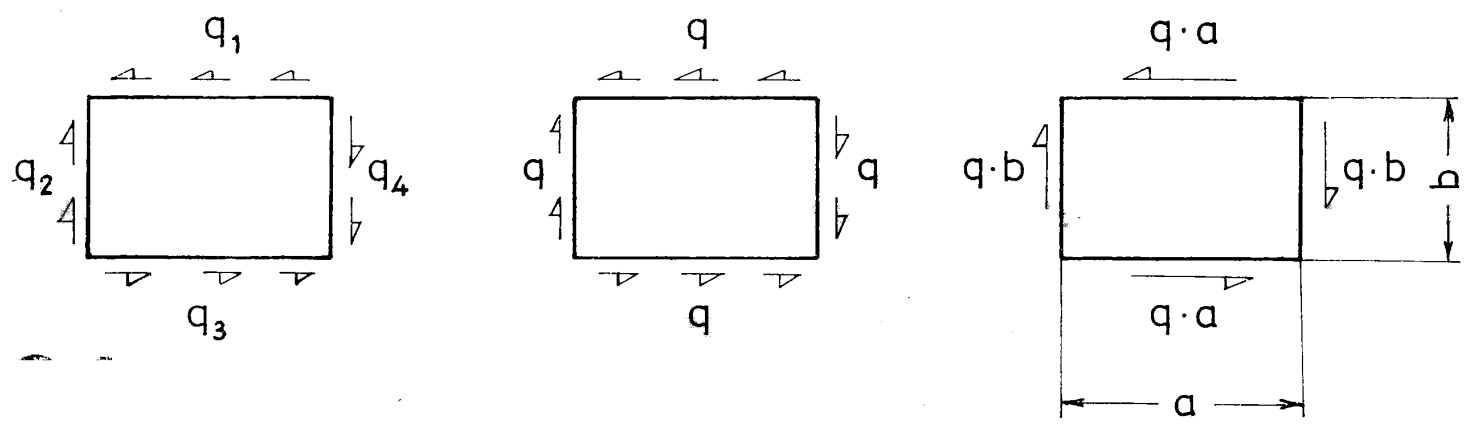


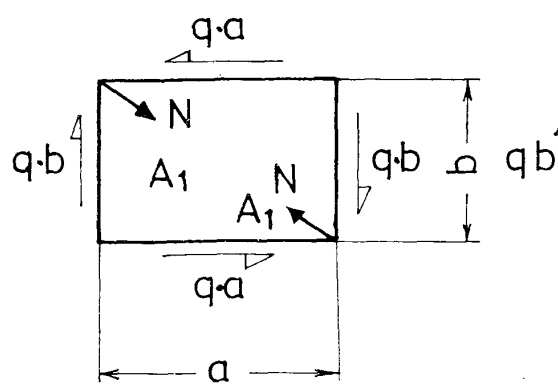

a)

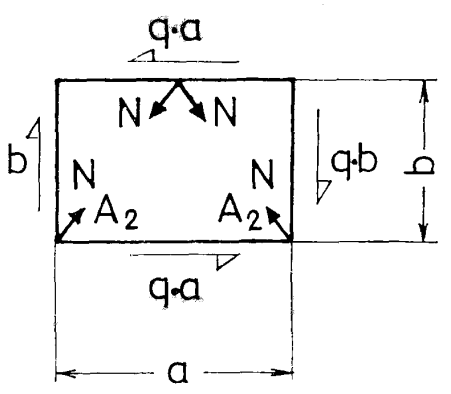

b)

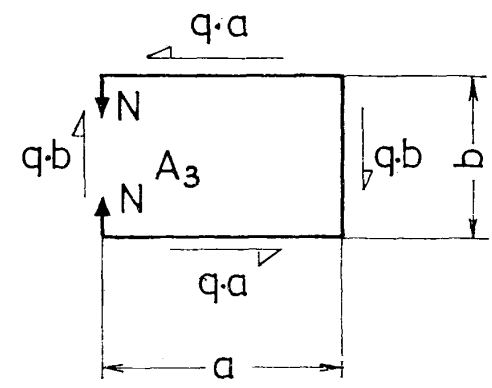

c)

La expresión de la energía potencial de deformación de deslizamiento del alma de espesor $t_{e q}$ es:

$$
U_{1}=\frac{q^{2} \cdot a \cdot b}{2 \cdot G \cdot t_{e q}}
$$

— La de una diagonal (fig. 25 a):

$$
U_{2}=\frac{1}{2} \cdot N^{2} \cdot \frac{\sqrt{a^{2}+b^{2}}}{E \cdot A_{1}}=\frac{1}{2} \cdot q^{2} \cdot \frac{\left(a^{2}+b^{2}\right)^{3 / 2}}{E \cdot A_{1}}
$$

— La de dos diagonales (fig. $25 \mathrm{~b}$ ):

$$
U_{3}=N^{2} \cdot \frac{\sqrt{0,25 \cdot a^{2}+b^{2}}}{E \cdot A_{2}}=q^{2} \cdot \frac{\left(0,25 \cdot a^{2}+n^{2}\right)^{3 / 2}}{E \cdot A_{2}}
$$

- La de un montante (fig. 25 c):

$$
U_{4}=\frac{N^{2} \cdot b}{2 \cdot E \cdot A_{3}}=\frac{q^{2} \cdot b^{2}}{2 \cdot E \cdot A_{3}}
$$

- Y por fin la de un marco (fig. 26):

$$
U_{5}=0,5 \cdot \sum \frac{1}{E \cdot J} \int M^{2} \cdot d x=\frac{q^{2} \cdot a^{2} \cdot b^{2}}{48 \cdot E}\left(\frac{a}{J_{a}}+\frac{b}{J_{b}}\right)
$$

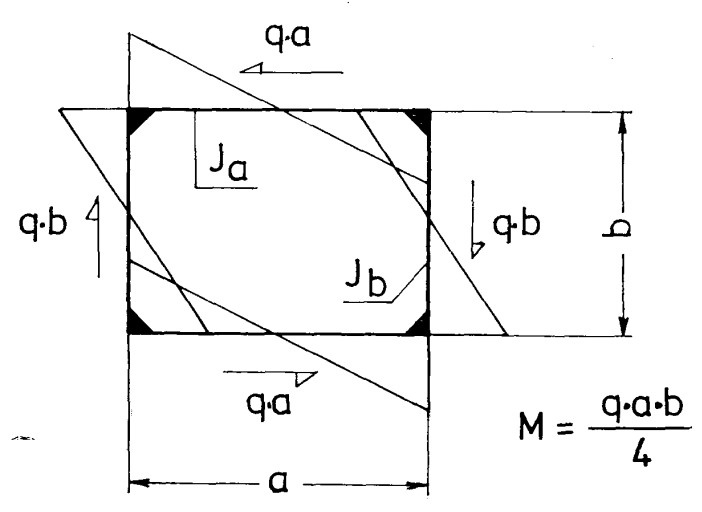

Igualando los valores de $U_{1}$ de la [45] a los segundos miembros de las expresiones de la energía de las correspondientes almas en celosía, o aporticadas, encontramos el espesor del alma llena equivalente.

Así, de la condición $U_{1}=U_{2}+U_{4}$ obtenemos:

- Para el alma con triangulación Pratt:

$$
t_{e q}=\frac{E \cdot a \cdot b}{G \cdot\left[\frac{\left(a^{2}+b^{2}\right)^{3 / 2}}{A_{1}}+\frac{b^{3}}{A_{3}}\right]}
$$


- Para el alma con triangulación Warren sin montantes:

-- Y para el marco simple:

$$
t_{e q}=\frac{E \cdot A_{1} \cdot a \cdot b}{G \cdot\left(a^{2}+b^{2}\right)^{3 / 2}}
$$

$$
t_{e q}=\frac{24 \cdot E}{G \cdot a \cdot b \cdot\left(\frac{a}{J_{a}}+\frac{b}{J_{b}}\right)}
$$

Cuando el alma es de paneles (células) múltiples (figs. 27 y 28), al pertenecer cada uno de los montantes a dos paneles contiguos, en la [50] debemos introducir $0,5 \cdot A_{3}$ en lugar de $A_{3}$, y en la [52] en lugar de $J_{b}$ introduciremos $0,5 \cdot J_{b}$.
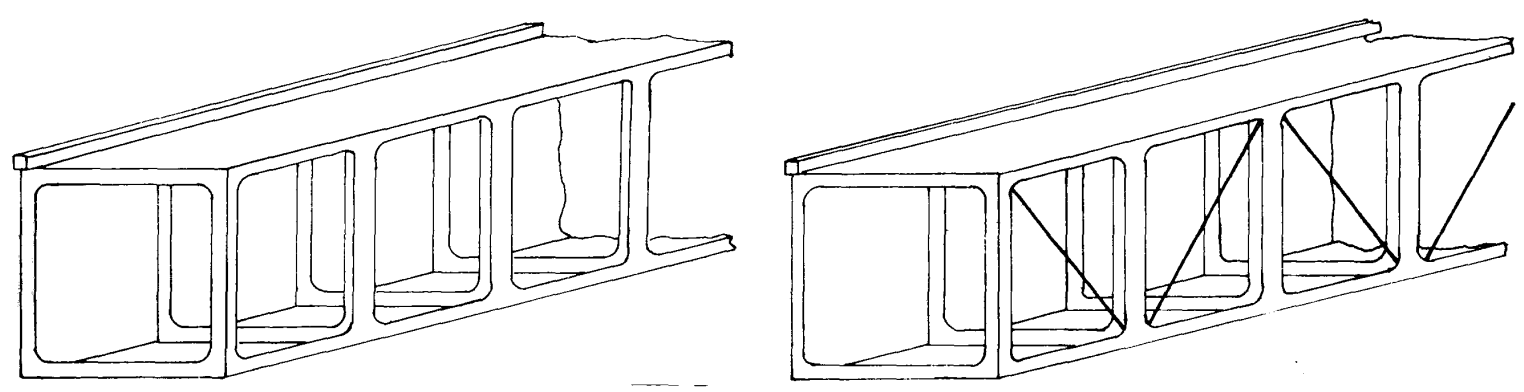

Excluyendo las naves de los grandes complejos siderúrgicos, servidas por puentes-grúa de capacidades de elevación muy importantes, donde las vigas-carriles salvan grandes luces, en el resto de la edificación industrial no son corrientes las vigas-carriles en cajón con cuatro, incluso tres, caras de alma llena. El caso más normal es el de sólo dos caras en alma llena; pero los procedimientos prácticos de cálculo del espesor del alma equivalente y del centro de torsión de la pieza expuestos, tanto para el perfil cerrado como el abierto, siguen siendo válidos.

Aquí ponemos punto final, con la esperanza de que con el material presentado, quizá, hayamos podido contribuir a la divulgación del funcionamiento de las vigas-carriles y ayudado en algo al proyectista de estructuras metálicas.

\section{REFERENCIAS}

1. Rivacoвa, J.: «Sobre el funcionamiento de la viga-carril y las tensiones locales y secundarias». Primera parte. Informes de la Construcción, núm. 265, 1974.

2. LAMPSI, B. B.: «Napriazhonnoye sostoyañiye polosy pri odnom iz sluchayev vozdieystviya kasatiel'nykh usiliy na yeyo kromku" (Estado tensional de una llanta bajo uno de los casos de acción de los esfuerzos tangenciales sobre su borde). Dierieviannyie y Mietallischeskiye Konstruktsii, núm. 45, 1964.

3. OdIN, I. M.: «Inzheniernyie zadachi raschota mietallokonstrutsii» (Problemas de ingeniería en el cálculo de las estructuras metálicas). Mashguiz, 1972.

4. Timoshenko, S.; Woinowsky-KrIEger, S.: «Teoría de placas y láminas». Ediciones Urmo, 1970.

5. KAHN, S. N.: «Raschot tonkostiennykh aviakonstruktsii» (Cálculo de las estructuras de pared delgada del avión). Oboronguiz, 1948.

6. Parñitsky, A. B.; Shabashov, A. P.: «Mostovyie Krany obschiego naznacheñiya» (Puentes-grúa de destino general). Mashguiz, 1958.

7. HochBERG, M. M.: «Mietallicheskiye konstruktsii podyomno-transportnykh mashin» (Estructuras metálicas de las máquinas de elevación y transporte). Mashguiz, 1969.

8. Vlasov, B. Z.: «Pièces longues en voiles minces». Eyrolles, 1962.

9. Feodósiev, V. I.: «Resistencia de Materiales». Editorial Mir, 1972.

10. Chevallon, J.: «La estructura metálica de la acería Solmer». Acier-Stahl-Steel, n. 11-1974. 


\section{résumé}

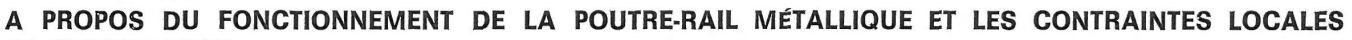
ET SECONDAIRES

José Rivacoba, ingénieur des Ponts et Chaussées,

Chef de la Section de Structures Métalliques de SENER

La deuxième partie de l'article complète l'information sur la poutre-rail en double $\mathrm{T}$, présentée dans la première partie de l'article, et offre des commentaires sur les métodes analytiques de calcul de la poutre-rail en caisson.

\section{summary}

REGARDING THE FUNCTIONING OF THE METAL DOUBLE T RAIL GIRDER AND THE LOCAL AND SECONDARY STRESSES

José Rivacoba, civil engineer,

Head of the Metal Structures Section of SENER

The second part of the article completes the information regarding the double $T$ rail girder, presented in the first part of the article and it comments upon the analytical calculus methods of the box girder for a travelling crane.

\section{zusammenfassung}

ÜBER DIE FUNKTION DES METALLISCHEN BRUCKENTRÄGERS UND DIE LOKALEN UND SEKUNDÄREN SPANNUNGEN

José Rivacoba, Zivilingenieur,

Chef des Metallstruktursektion in SENER

Die zweïte Hälfte des Artikels ergänzt die Information über den in dem ersten Teil des Artikels präsentierten Doppel-T-Brückenträger und es werden die analythischen Berechnungsmethoden des rektangularen Bruckenträgers diskutiert.

\section{publicaciones del i. e. t. c. c.}

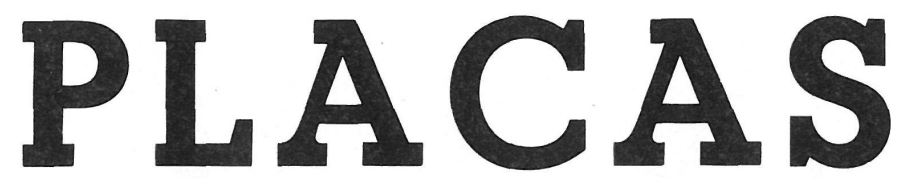

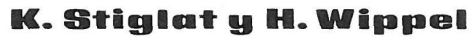
Drs. Ingenieros

Traducción de Juan Batanero

Dr. Ingeniero de Caminos

con la colaboración de

Francisco Morán

Ingeniero de Caminos

Este libro, cuidadosa y magníficamente editado, reúne, quizás, la más completa colección conocida de tablas para placas, por los numerosos casos de vinculación y de carga estudiados y por la abundancia de relaciones de dimensión y de datos ofrecidos, que cubren prácticamente todo el campo de las losas en edificación. Permite desarrollar, con comodidad, rapidez y una aproximación suficiente, los cálculos de dimensionamiento y comprobación, obviando las dificultades que como es sabido, presenta el desarrollo numérico de los métodos de cálculo de estos elementos, evitando enojosas operaciones.

Trata la obra sobre "Zonas de Placas", "Placas sobre apoyos puntuales", "Placas apoyadas en dos, tres y cuatro bordes" y "Placas apoyadas elásticamente", tipos que en la actualidad disponían de una documentación, íncompleta o nula, para la determinación de esfuerzos. Los corrimientos de la placa, como valores previos para la determinación de los momentos, han sido obtenidos por medio del Cálculo de Diferencias, método que se ha comprobado como suficientemente satisfactorio, aún en su forma simple, aplicado con un cierto control.

Un volumen encuadernado en tela, de $30,5 \times 23,5 \mathrm{~cm}$, compuesto de 92 págs. Madrid, 1968.

Precios: España, 925 ptas.; extranjero, \$18.50. 